TRANSACTIONS OF THE

AMERICAN MATHEMATICAL SOCIETY

Volume 356, Number 2, Pages 703-733

S 0002-9947(03)03213-6

Article electronically published on August 25, 2003

\title{
SOME PICARD THEOREMS FOR MINIMAL SURFACES
}

\author{
FRANCISCO J. LÓPEZ
}

\begin{abstract}
This paper deals with the study of those closed subsets $F \subset \mathbb{R}^{3}$ for which the following statement holds:

If $S$ is a properly immersed minimal surface in $\mathbb{R}^{3}$ of finite topology that is eventually disjoint from $F$, then $S$ has finite total curvature.

The same question is also considered when the conclusion is finite type or parabolicity.
\end{abstract}

\section{INTRODUCTION}

By definition, a surface has finite topology if it is homeomorphic to a connected compact surface (with possibly non-empty boundary) minus a finite set of interior points.

A Riemann surface with non-empty boundary is said to be parabolic if bounded harmonic functions are determined by their boundary values. However, in the context of non-compact Riemann surfaces with empty boundary, parabolicity has a different meaning: the surface does not carry a negative non-constant subharmonic function. For more details, see [1] and [16]. A compact Riemann surface without boundary is called elliptic, and a Riemann surface with possibly non-empty boundary which is neither elliptic nor parabolic is called hyperbolic. A non-hyperbolic Riemann surface of finite topology is called of finite conformal type. It is not hard to see that finite conformal type means that the surface is conformally equivalent to a compact Riemann surface (with possibly non-empty boundary) minus a finite set of interior points.

Let $X: M \rightarrow \mathbb{R}^{3}$ be a complete minimal immersion, and label $(g, \eta)$ as its Weierstrass representation (that is to say, $X=\frac{1}{2}$ Real $\left.\int\left(\left(1-g^{2}\right) \eta, i\left(1+g^{2}\right) \eta, 2 g \eta\right)\right)$. By definition, $X$ is of finite type if and only if $M$ is of finite conformal type, and up to a rigid motion, $\eta g$ and $\frac{d g}{g}$ are meromorphic 1-forms on the compactification of $M$, 24.

Complete minimal surfaces of finite total curvature are of finite type 21, but the contrary is false: the helicoid is a counterexample. By definition, two closed subsets of $\mathbb{R}^{3}$ are eventually disjoint if they do not intersect outside a compact set.

In this paper we are going to consider some Picard type problems for properly minimal surfaces of finite topology in $\mathbb{R}^{3}$. More precisely, let $\mathcal{P}_{0}$ (respectively, $\mathcal{P}_{1}$ and

Received by the editors November 29, 2001 and, in revised form, September 17, 2002.

2000 Mathematics Subject Classification. Primary 53A10; Secondary 53C42.

Key words and phrases. Properly immersed minimal surfaces, finite topology, finite total curvature.

The author's research was partially supported by MCYT-FEDER grant number BFM20013489 .

(C)2003 American Mathematical Society 
$\mathcal{P}_{2}$ ) be the space of properly immersed minimal surfaces in $\mathbb{R}^{3}$ of finite conformal type (respectively, finite type and finite total curvature). Our interest is in studying those closed subsets $F \subset \mathbb{R}^{3}$ for which the following statements hold:

Statement $j(=0,1,2)$. The space of properly immersed minimal surfaces in $\mathbb{R}^{3}$ of finite topology and eventually disjoint from $F$ lies in $\mathcal{P}_{j}$.

Obviously, if Statement $j$ holds, so does Statement $i, i \leq j$, but Statement $h$ need not be valid for $h>j$.

It is known that Statement 2 holds when $F$ is a shallow enough double cone (the cone lemma [9]), and when $F$ is a closed half space and the surfaces have no boundary (the strong half space theorem [10]). In the second case, the surfaces must be planes.

In the embedded case, Statement 2 holds for $F=\emptyset$ and surfaces with more than one end [17], 4]. It is also valid when $F$ is a sufficiently narrow downward sloping cone and the number of ends of the surface is arbitrary [5].

Statement 1 holds for $F=\emptyset$ and embedded surfaces with one end and empty boundary [19].

Finally, Statement 0 is valid for $F$ a closed half space and arbitrary immersed surfaces [6]. Very recently, Morales 20] proved that Statement 0 is not valid for $F=\emptyset$, disproving a conjecture by Sullivan.

The main goal of this paper is showing some new closed subsets for which the Main Statement is valid (see Figure 1).

The following results have been established:

(a) If $F$ is a suitable collection of truncated non-compact planar sectors, then Statement 2 holds. Roughly speaking, the sectors in F must satisfy the following conditions: they have the same angle, they are contained in pairwise non-parallel planes, they are pairwise disjoint, they are transverse to a double cone, they are homogeneously distributed in $\mathbb{R}^{3}$, the convex hull of the collection is $\mathbb{R}^{3}$, and the number of sectors is large enough in terms of their angle. See Definition 4.1. Figure 4 and Theorem 4.1 for details.

The definition and construction of these families of planar sectors strongly depend on the non-existence results stated in Theorem 2.5 .

(b) If $F$ consists of three disjoint and vertical half planes not contained in a half space, then Statement 2 holds. Furthermore, the surfaces disjoint from $F$ have only planar ends with horizontal limit normal vectors. See Theorem 4.2 and Corollary 4.4 .

(c) If $F$ consists of three disjoint and vertical half planes and $F$ is not contained in a wedge of angle less than $\pi$ of $\mathbb{R}^{3}$, then Statement 0 holds. Moreover, Statement 2 holds for surfaces with bounded curvature. See Theorem 4.3 and Corollary 4.5

(d) If $F$ consists of the planar complements of three city maps in parallel planes, then Statement 0 holds. Moreover, Statement 1 holds for surfaces with bounded curvature.

Roughly speaking, this result is valid when the city maps are parallel and the width of their streets is less than the distance between the planes containing them. See Theorem 4.4 for details. 


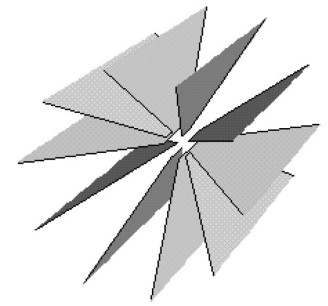

(a)

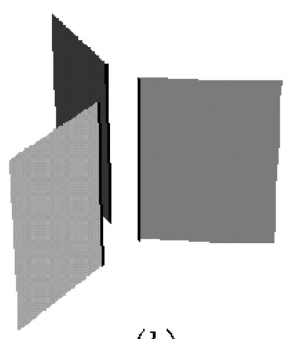

$(b)$

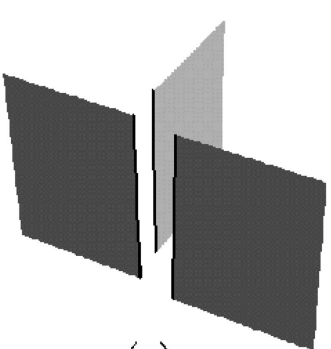

(c)

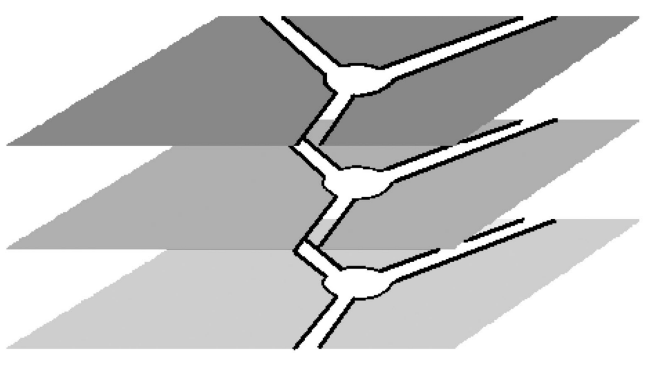

$(d)$

Figure 1. (a) A collection of planar sectors. (b) Three vertical half planes with convex hull $\mathbb{R}^{3}$. (c) Three half planes with convex hull a half space. $(d)$ The planar complements of three parallel city maps.

Furthermore, if we do not assume that the curvature is bounded, then Statement 1 fails for closed subsets like those in $(c)$ and $(d)$, and the same occurs for Statement 2 when $F$ is a closed half space. See Remark 4.1.

The key step in the proof of these results is the existence of finite planes, that is to say, planes splitting the surface into a finite number of connected components. This fact guarantees that the surface is parabolic 18 and yields interesting information about the Gauss map of the surface. So, we can prove:

If a properly immersed minimal surface $S$ of finite topology has a finite plane $\Sigma$, then the Gauss map of $S$ takes on the two normal vectors of $\Sigma$ a finite number of times. As a consequence, $S$ has finite total curvature if and only if it has two non-parallel finite planes (see Theorem 3.1 and Corollary [3.1).

The existence of finite planes has been basically derived from two ingredients: from the non-existence results of properly immersed minimal surfaces with planar boundary in truncated tetrahedral domains stated in Theorem 2.5 and Corollary 4.3, and from Lemma 4.2, which is devoted to the geometry of properly immersed minimal discs in a wedge of $\mathbb{R}^{3}$.

Finally, and as a consequence of all the above ideas, the following characterizations of the plane have been obtained:

The plane is the only properly immersed minimal surface in $\mathbb{R}^{3}$ of finite topology and empty boundary satisfying any of the following conditions: 
(i) There exist two planes meeting the surface in a straight line (which depends on the plane). See Corollary [3.2.

(ii) The surface has only one end, and is disjoint from a closed subset $F$ as in (b). See Corollary 4.4.

(iii) The surface has bounded curvature, only one end, and is disjoint from a closed subset $F$ as in (c). See Corollary 4.5 .

Concerning $(i)$, note that we have not assumed that the surface is embedded, and so the straight lines in $(i)$ could be multiple. Furthermore, the same result is false if we only assume that there is one plane meeting the surface in a straight line, even in the finite total curvature case. See Figure 13 for a counterexample. Some closely related results can be also found in [3] and [25].

The paper is laid out as follows. In Section 2, we introduce some notation and state some known results. In Section 3 we study the Gauss map of properly immersed minimal surfaces of finite topology having finite planes. In Subsection 3.1 we study the relationship between finite planes and finite total curvature. The deepest results lie in Section 4 where the asymptotic behavior of properly immersed minimal surfaces with finite topology is studied. So, $(a)$ and $(b)$ are proved in Subsections 4.1 and 4.2, respectively, while $(c)$ and $(d)$ are proved in Subsection 4.3 .

\section{Notation AND PRELiminaries}

By definition, a simple arc in a surface $M$ is a properly embedded curve in $M$ homeomorphic to an interval. As usual, $\mathbb{S}^{n-1}(R)=\left\{x \in \mathbb{R}^{n}:\|x\|=R\right\}, R>0$, and if $R=1$, we simply write $\mathbb{S}^{n-1}=\mathbb{S}^{n-1}(1), n \geq 2$.

Let $A \subset \mathbb{R}^{3}$ be a non-compact subset, and let $A[R]$ be the homothetical shrinking

$$
\frac{1}{R} \cdot\left(A \cap \mathbb{S}^{2}(R)\right) \stackrel{\text { def }}{=}\left\{\frac{1}{R} \cdot P: P \in A \cap \mathbb{S}^{2}(R)\right\},
$$

$R>1$. Assume that $\lim _{R \rightarrow+\infty} A[R]=A_{0} \subset \mathbb{S}^{2}$ in the Hausdorff sense, i.e.,

$$
A_{0}=\left\{p \in \mathbb{S}^{2}: \liminf _{R \rightarrow \infty} d(p, A[R])=0\right\} .
$$

Then, we refer to $A_{0}$ as the base of $A$, and write $\mathcal{B}(A)=A_{0}$.

Let $A_{1}$ and $A_{2}$ be subsets of $\mathbb{R}^{3}$ for which the base is well defined, and assume there are an open domain $\Omega$ in $\mathbb{S}^{2}$ and a real number $R_{0}>1$ such that $\mathcal{B}\left(A_{1}\right) \subset$ $\Omega \subset \bigcap_{R \geq R_{0}} A_{2}[R]$. Then, it is easy to check that, up to a compact subset,

$$
A_{1} \subset A_{2} .
$$

We will need the following theorems:

Theorem 2.1 (Jorge and Meeks [12]). Let $\left.\left.X: A \rightarrow \mathbb{R}^{3}, A \cong\right] 0,1\right] \times \mathbb{S}^{1}$, be a complete immersed minimal annulus with finite total curvature. Let $\Sigma$ denote the only plane passing through the origin and orthogonal to the limit normal vector of $X(A)$ at infinity, and let $\mathfrak{p}: X(M) \rightarrow \Sigma$ be the orthogonal projection of $X(M)$ on $\Sigma$.

Then, $\mathfrak{p}$ is proper, and, up to removing a compact subset, $X(A)$ is a multi-sheeted graph of sublinear growth over $\Sigma$. Furthermore, the base of $X(A)$ is well defined, and $\mathcal{B}(X(A))=\mathbb{S}^{2} \cap \Sigma$. 
By definition, the multiplicity of the annular end is the number of sheets of $\mathfrak{p}$, that is to say, the winding number of $\mathcal{B}(X(A))$ as limit curve. So, the end $X(A)$ is eventually embedded if and only if the multiplicity is equal to one.

Theorem 2.2 (Fang and Meeks [7]). Let $X: M \rightarrow \mathbb{R}^{3}$ be a properly immersed minimal surface contained in a half space $H$, and assume that $X(\partial(M)) \subset \partial(H)$. Then,

$$
\text { Supremum }\{d(X(P), \partial(H)): P \in M\}=+\infty,
$$

where $d$ means Euclidean distance.

The following definition will be useful.

Definition 2.1. Let $X: M \rightarrow \mathbb{R}^{3}$ be a properly immersed minimal surface in $\mathbb{R}^{3}$, and let $\Sigma$ be a plane in $\mathbb{R}^{3}$. We say that $\Sigma$ is a finite plane for $X$ (and that $X$ has $\Sigma$ as finite plane) if, up to removing a compact subset of $M$, the set $X^{-1}(X(M)-\Sigma)$ is empty or contains finitely many connected components.

We have

Theorem 2.3 (Meeks and Rosenberg [18]). Let $X: M \rightarrow \mathbb{R}^{3}$ be a properly immersed minimal surface with finite topology. If $X$ has a finite plane, then $M$ is of finite conformal type.

Given $A \subset \mathbb{R}^{3}$, the convex hull of $A$ will be denoted by $\mathcal{E}(A)$. The symbol $\perp$ means orthogonal, and $\|$ means parallel.

Let $C$ be a right solid cylinder over a compact planar domain, and let $\Sigma$ be a plane transversal to $C$, that is to say, meeting $C$ in a compact set. Let $\Sigma^{+}$and $\Sigma^{-}$ be the closed half spaces bounded by $\Sigma$. By a truncated solid cylinder we mean any closed subset of $C$ eventually disjoint from $\Sigma^{-}$and containing $C \cap \Sigma^{+}$.

A wedge $W$ of $\mathbb{R}^{3}$ is the non-void intersection of two closed half spaces $H$ and $H^{\prime}$ with non-parallel boundary planes. The planes $\partial(H)$ and $\partial\left(H^{\prime}\right)$ make in $W$ an angle $a(W) \in] 0, \pi[$. Slabs and half spaces can be considered as wedges of angles 0 and $\pi$, respectively. If $a(W) \in] 0, \pi\left[\right.$, the straight line $l(W)=\partial(H) \cap \partial\left(H^{\prime}\right)$ is the axis of $W$. If $a(W)=0$ (resp., $a(W)=\pi$ ), an axis $l(W)$ of $W$ is any straight line in the only plane which is parallel to $\partial(H)$ and bisects the slab $W$ (resp., any straight line in $\partial(W))$. If $a(W)>0$, the bisector plane of $W$ is the plane $\Pi(W)$ containing $l(W)$ and splitting $W$ into two pieces symmetric with respect to $\Pi(W)$. If $W$ is a slab, $\Pi(W)$ is the plane parallel to $W$ and bisecting it. The plane $\Pi(W)$ is uniquely determined, except when $a(W)=\pi$, in which case $\Pi(W)$ depends on the axis $l(W)$. If $W$ is a wedge and $\mathcal{C}$ is a solid circular cylinder with axis parallel to $l(W)$ and meeting $W$, then $\overline{\mathcal{E}(W-\mathcal{C})}$ is said to be a truncated wedge.

Given a plane $\Pi \subset \mathbb{R}^{3}$, a domain $S$ in $\Pi$ is said to be a sector if $S$ is the intersection of $\Pi$ and a wedge $W$ whose axis is not parallel to $\Pi$. If $0<a(W)<\pi$, the angle made in $S$ by the two half-lines in $\partial(S)$ will be denoted by $a(S)$. By definition, strips and half planes are sectors of angles 0 and $\pi$, respectively.

We will need the following result:

Theorem 2.4 ([15]). Let $S$ be a properly immersed minimal surface in $\mathbb{R}^{3}$ contained in a wedge $W$ of angle less than $\pi$, and assume there exists a half space $H$ such that $\partial(S) \subset H$ and $\partial(H)$ is not parallel to $l(W)$.

Then $S \subset \mathcal{E}(\partial(S))$. 
Let $W$ and $W^{\prime}$ be two wedges satisfying $a(W) \in\left[0, \pi\left[, \Pi(W)=\left\{x_{3}=0\right\}\right.\right.$, $\left.l(W) \perp\left\{x_{2}=0\right\}, a\left(W^{\prime}\right) \subset\right] 0, \pi\left[, \Pi\left(W^{\prime}\right)=\left\{x_{2}=0\right\}, l\left(W^{\prime}\right) \perp\left\{x_{3}=0\right\}\right.$, and $\left\{\left(x_{1}, 0,0\right): x_{1} \geq 0\right\} \subset W \cap W^{\prime}$. Consider the truncated tetrahedral domain $C=W \cap W^{\prime} \cap\left\{x_{1} \geq 0\right\}$. Then, denote by $F_{1}(C), F_{2}(C)$ the two faces of $\partial(C)$ in $\partial(W)$; by $F^{+}(C), F^{-}(C)$ the two faces of $\partial(C)$ in $\partial\left(W^{\prime}\right)$; and by $F_{0}(C)$ the face of $\partial(C)$ in $\left\{x_{1}=0\right\}$. Only $F_{0}(C)$ is compact, and it consists of either a rectangle, or a segment, or a point. Moreover, denote by $\mathfrak{h}(C)$ and $\mathfrak{o}(C)$ the height and the width of the base of $C$, respectively. We also set $\vartheta(C) \stackrel{\text { def }}{=} a(W)$ and $\varrho(C) \stackrel{\text { def }}{=} a\left(W^{\prime}\right)$. See Figure 2 for details. We call $\partial\left(F^{+}(C)\right)$ the polygonal boundary of $F^{+}(C)$ as planar domain, and in a similar way, we define $\partial\left(F^{-}(C)\right)$. Finally, set $\Upsilon(C) \stackrel{\text { def }}{=} \partial\left(F^{+}(C)\right) \cup \partial\left(F^{-}(C)\right)$.

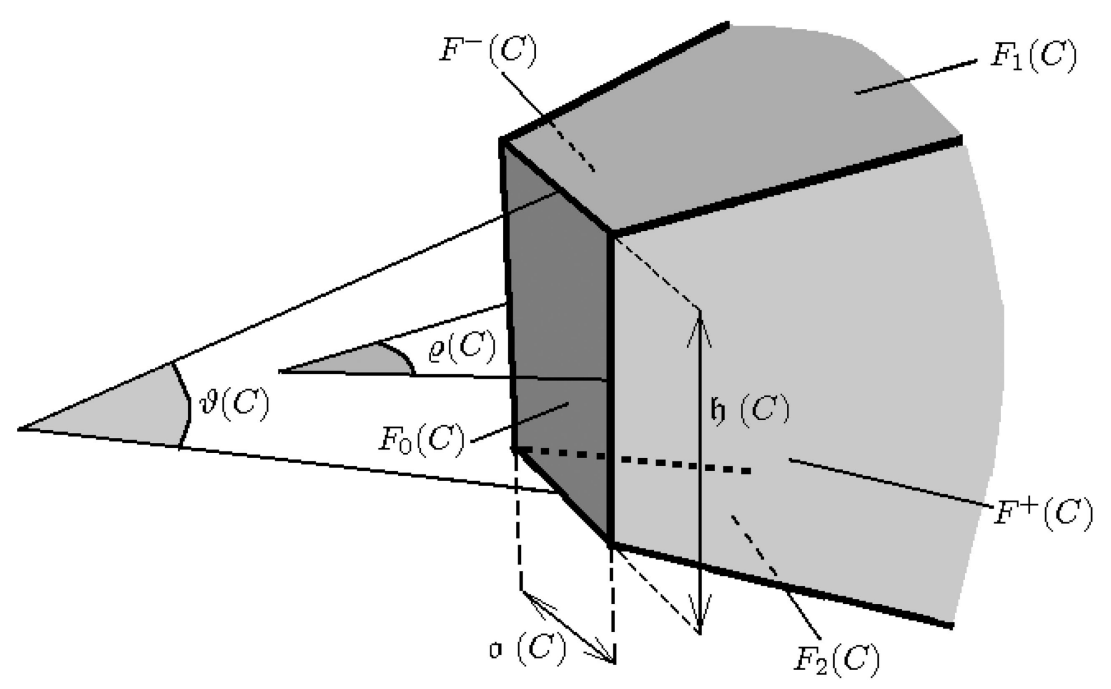

Figure 2. The domain $C$.

A deep study of the domains $C$ as above which admit a minimal surface spanning $\Upsilon(C)$ can be found in [15]. These surfaces can be used, in an elaborate way, as barriers for the maximum principle application, leading to some non-existence results for non-flat minimal surfaces $S \subset C$ whose boundary lies in the vertical faces $F^{+}(C)$ and $F^{-}(C)$ :

Theorem 2.5 ([15]). There exist an increasing analytical diffeomorphism

$$
\left[0, \pi\left[\rightarrow \left[0, \pi\left[, \quad \theta \rightarrow \rho_{\theta},\right.\right.\right.\right.
$$

and a positive continuous map

$$
\mathcal{A} \rightarrow] 0,+\infty\left[, \quad(\theta, \rho) \rightarrow o_{\theta, \rho},\right.
$$

where $\mathcal{A}=\left\{(\theta, \rho): \theta \in\left[0, \pi[, \rho \in] \rho_{\theta}, \pi[\}\right.\right.$, such that:

(a) If $(\theta, \rho),\left(\theta^{\prime}, \rho^{\prime}\right) \in \mathcal{A}$ and $\theta \geq \theta^{\prime}, \rho \leq \rho^{\prime}$, then $o_{\theta, \rho} \geq o_{\theta^{\prime}, \rho^{\prime}}$.

(b) If $C$ is a domain as in Figure $[$ such that $\mathfrak{h}(C)=1,(\vartheta(C), \varrho(C)) \subset \mathcal{A}$, $\mathfrak{o}(C)>o_{\vartheta}(C), \varrho(C)$, and $S$ is a connected properly immersed minimal surface 
in $\mathbb{R}^{3}$ contained in $C$ and with boundary $\partial(S)$ lying in $F^{+}(C) \cup F^{-}(C)$, then $S$ is a planar domain (lying in either $F^{+}(C)$ or $F^{-}(C)$ ).

\section{Finite planes And the Gauss map}

This section is devoted to the study of the Gauss map of properly immersed minimal surfaces with finite topology. So, we are going to prove that the normal vector of a finite plane is taken by the Gauss map a finite number of times. This result was inspired by some nice ideas in [7].

First of all, we introduce the following notation.

Let $\Sigma_{0}$ be the plane $\left\{x \in \mathbb{R}^{3}:\langle x, v\rangle=0\right\}$, where $v$ is a non-zero vector and $\langle$,$\rangle is the Euclidean metric in \mathbb{R}^{3}$. Define $\Sigma_{t}=\left\{x \in \mathbb{R}^{3}:\langle x, v\rangle=t\right\}$, $\Sigma_{t}^{+}=\left\{x \in \mathbb{R}^{3}:\langle x, v\rangle>t\right\}$ and $\Sigma_{t}^{-}=\left\{x \in \mathbb{R}^{3}:\langle x, v\rangle<t\right\}, t \in \mathbb{R}$.

Let $X: A \rightarrow \mathbb{R}^{3}$ be a properly immersed minimal annulus, $\left.\left.A \cong\right] 0,1\right] \times \mathbb{S}^{1}$, and let $N: A \rightarrow \mathbb{R}^{3}$ be its Gauss map.

Write $A_{t}=X^{-1}\left(\Sigma_{t}\right), A_{t}^{+}=X^{-1}\left(\Sigma_{t}^{+}\right)$and $A_{t}^{-}=X^{-1}\left(\Sigma_{t}^{-}\right)$. The nodal set $A_{t}$ consists of a family of properly immersed analytic curves in $A$. If $P \in A_{t} \cap$ $N^{-1}(\{v,-v\})$, then there is a cross of higher order singularity in $A_{t}$ at $P$. In fact, if the Gauss curvature of $X(A)$ at $P$ does not vanish, then in $A_{t}$ near $P$ there are two curves that cross orthogonally at $P$; if the Gauss curvature vanishes, and the multiplicity of the Gauss map at $P$ is $k \geq 2$, then, near $P, A_{t}$ consists of $k+1$ curves that cross at equal angles at $P$. In the following, we denote by $V_{t}$ the set $A_{t} \cap N^{-1}(\{v,-v\})$, and the points of $\bigcup_{t \in \mathbb{R}} V_{t}$ are called $v$-points.

The connected components of $A-A_{t}$ (i.e., the connected components of $A_{t}^{+}$and $A_{t}^{-}$) will be called regions of $A-A_{t}$. By the maximum principle, for two regions of $A-A_{t}$ which have an arc in $A_{t}$ as part of their common boundary, one lies in $A_{t}^{+}$ and the other lies in $A_{t}^{-}$.

Let $P \in V_{t}$, and take a small disk $D \subset A$ centered at $P$ such that $D \cap V_{t}=\{P\}$ and $A_{t} \cap D$ divides $D$ into at least four components, each of them having $P$ in its boundary. Among these components, there are two in $A_{t}^{+}$and two in $A_{t}^{-}$. We say an embedded curve $\gamma$ in $A_{t}^{+} \cup V_{t}$ (resp., $A_{t}^{-} \cup V_{t}$ ) passes through $P \in V_{t}$ if $P \in \gamma$ and the component of $\gamma \cap D$ which contains $P$ crosses two distinct components of $D \cap A_{t}^{+}$(resp., $D \cap A_{t}^{-}$). Hence, if $\gamma \subset A_{t}^{+} \cup V_{t}$ (resp., $\gamma \subset A_{t}^{-} \cup V_{t}$ ) is an embedded loop passing through $P \in V_{t}$, then the two connected components of $A-\gamma$ contain a region of $A_{t}^{-}$(resp., $A_{t}^{+}$).

We need the following lemma.

Lemma 3.1. Let $t_{0} \in \mathbb{R}$, and assume that either $A_{t_{0}}^{-}$is empty or it has finitely many regions. Then, up to removing a compact piece of $A$, either $A_{t}^{-}$is empty or it has finitely many regions, $t \geq t_{0}$. Moreover, the set $\bigcup_{t \geq t_{0}} V_{t}$ is finite.

Proof. Up to removing a compact subset of $A$, we can assume that $\partial(A)$ is analytic, $\pm v \notin N(\partial(A))$, and the collection of points $B$ in $\partial(A)$ whose tangent line is orthogonal to $v$ is a finite set. Hence, it is not hard to see that $\sharp\left(A_{t} \cap \partial(A)\right) \leq \sharp(B)$, $t \in \mathbb{R}$.

Let $\Omega$ be a region of $A-A_{t}, t \in \mathbb{R}$. During the proof, $\partial(\Omega)=(\bar{\Omega}-\Omega) \cup(\partial(A) \cap \bar{\Omega})$. By the maximum principle, $\overline{\partial(\Omega)-\partial(A)}$ does not contain any homotopically trivial embedded loop. Thus, the domains $\Omega$ such that $\partial(\Omega) \subset A_{t}$ are not compact, and from Theorem [2.2, Supremum $\left\{\operatorname{dist}\left(X(P), \Sigma_{t}\right): P \in \Omega\right\}=+\infty$. 
Therefore: $(i)$ if $\Omega$ contains an embedded loop homotopic to $\partial(A)$, then either $\bar{\Omega}$ is homeomorphic to a closed annulus minus a (maybe empty) set of boundary points, or it is homeomorphic to a closed disk minus an interior point; (ii) if $\bar{\Omega}$ does not contain an embedded loop homotopic to $\partial(A)$, then $\bar{\Omega}$ is homeomorphic to a closed disk minus a (maybe empty) set of boundary points; and (iii) if $\bar{\Omega}$ contains an embedded loop homotopic to $\partial(A)$, but $\Omega$ does not, then $\Omega-\partial(A)$ is homeomorphic to an open disk and $\partial(\Omega)$ contains a $v$-point. In case $(i i i)$, the region $\Omega$ is said to be special, and there is a $v$-point $P \in \partial(\Omega)$ such that, for a small disk $D$ centered at $P, D \cap \Omega$ contains two connected components sharing $P$ as boundary point. Any $v$-point in $\partial(\Omega)$ with this property will be called special.

Claim 1. Let $t \in \mathbb{R}$, and suppose there is an embedded loop $\Gamma$ in $A_{t}^{-} \cup V_{t}$ passing through a point of $V_{t}$. Then, $\Gamma$ is homotopic to $\partial(A)$.

The loop $\Gamma$ bounds a compact region $R$ in $A$ containing points of $A_{t}^{+}$, and so $R$ contains a region of $A_{t}^{+}$with compact closure. By the maximum principle, $\Gamma$ is not homotopically trivial, and so it is homotopic to $\partial(A)$. This proves the claim.

Claim 2. Let $t \in \mathbb{R}$. Then, $A_{t}^{-} \cup V_{t}$ does not contain two embedded loops $\Gamma$, $\Gamma^{\prime}$ satisfying the following three conditions: $(i)$ they are homotopic to $\partial(A),(i i)$ $\Gamma \cap \Gamma^{\prime} \subset V_{t}$, and (iii) either $\Gamma^{\prime}$ passes through a point $P \in V_{t}-\Gamma$ or $\Gamma^{\prime}$ intersects a region of $A_{t}^{-}$which is disjoint from $\Gamma$.

Claim 2 has five consequences that we shall need: $(a)$ The intersection of the boundaries of two distinct connected components of $A_{t}^{-}$contains at most two $v$ points. (b) The boundary of a special region of $A_{t}^{-}$contains at most one special $v$-point. (c) If $A_{t}^{-}$contains a special region, then, for any $t^{\prime}>t, A_{t^{\prime}}^{-}$contains an embedded loop homotopic to $\partial(A)$. (d) If $A_{t}^{-}$contains an embedded loop homotopic to $\partial(A)$, then $A_{t}^{-}$contains no special region. (e) If $A_{t}^{-}$contains finitely many connected components, then $V_{t}$ is finite.

We reason by contradiction, and suppose there are two loops $\Gamma$ and $\Gamma^{\prime}$ satisfying (i), (ii) and (iii) in Claim 2. The loops $\Gamma$ and $\Gamma^{\prime}$ bound two compact domains $R$ and $R^{\prime}$, respectively, in $A$, and the open set $\left(\stackrel{\circ}{R^{\prime}}-R\right) \cup\left(\stackrel{\circ}{R}-R^{\prime}\right)$ contains a region of $A_{t}^{+}$bounded by curves in $A_{t}$, which contradicts the maximum principle.

Let us first establish $(a)$. Let $\Omega^{\prime}$ and $\Omega^{\prime \prime}$ be two distinct connected components of $A_{t}^{-}$, and suppose that $\partial\left(\Omega^{\prime}\right) \cap \partial\left(\Omega^{\prime \prime}\right) \cap V_{t}$ contains three distinct points $P_{1}, P_{2}$ and $P_{3}$. Let $\gamma^{\prime}$ and $\gamma^{\prime \prime}$ denote two embedded arcs in $\Omega^{\prime} \cup\left\{P_{1}, P_{2}\right\}$ and $\Omega^{\prime \prime} \cup\left\{P_{1}, P_{2}\right\}$, respectively, joining $P_{1}$ and $P_{2}$. The loop $\Gamma=\gamma^{\prime} \cup \gamma^{\prime \prime}$ passes through $P_{1}$ and $P_{2}$, and by Claim 1, it is homotopic to $\partial(A)$. Likewise, we can find a loop $\Gamma^{\prime}$ in $\Omega^{\prime} \cup \Omega^{\prime \prime} \cup\left\{P_{1}, P_{3}\right\}$ passing through $P_{1}$ and $P_{3}$ and homotopic to $\partial(A)$. Without loss of generality, we can suppose that $\Gamma \cap \Gamma^{\prime}=\left\{P_{1}\right\}$. Hence, $\Gamma$ and $\Gamma^{\prime}$ satisfy $(i),(i i)$ and (iii) in Claim 2, which is a contradiction.

To prove (b), let $\Omega^{\prime}$ be a special region of $A_{t}^{-}$. If $P \in \partial\left(\Omega^{\prime}\right) \cap V_{t}$ is a special $v$-point, there is an embedded loop $\Gamma$ in $\Omega^{\prime} \cup\{P\}$ passing through $P$, and by Claim $1, \Gamma$ is homotopic to $\partial(A)$. Suppose that $\partial\left(\Omega^{\prime}\right) \cap V_{t}$ contains another special $v$-point $P^{\prime}$. The same argument gives the existence of an embedded loop $\Gamma^{\prime}$ in $\Omega^{\prime} \cup\left\{P^{\prime}\right\}$ passing through $P^{\prime}$, homotopic to $\partial(A)$. Note that such loops $\Gamma$ and $\Gamma^{\prime}$ can be chosen disjoint. Therefore, $\Gamma$ and $\Gamma^{\prime}$ satisfy $(i),(i i)$ and (iii) in Claim 2, which is again a contradiction. 
To prove (c), assume that $\Omega$ is a special region. Since $\Omega$ contains a special $v$-point $P_{0}$ in its boundary, we can construct as above an embedded loop $\Gamma$ in $\Omega \cup\left\{P_{0}\right\}$ passing through $P_{0}$. From Claim 1, we deduce that $\Gamma$ is homotopic to $\partial(A)$. Then, note that $\Gamma \subset A_{t^{\prime}}^{-}, t^{\prime}>t$.

The proof of $(d)$ is similar to the proofs of $(a)$ and $(b)$.

Finally, to prove $(e)$, note that each point of $V_{t}$ lies in the boundary of a finite collection of connected components of $A_{t}^{-}$. So, if $\sharp\left(V_{t}\right)=\infty$, we can find two regions $\Omega^{\prime}$ and $\Omega^{\prime \prime}$ in $A_{t}^{-}$(which may be the same) containing infinitely many $v$-points in their common boundary, which contradicts either $(a)$ or $(b)$ and proves the claim.

In accordance with Claim 2, there exists at most one special region in $A_{t}^{-}$, $t \in \mathbb{R}$ : otherwise, reasoning as in the proof of $(b)$ in Claim 2, we can construct two embedded loops satisfying the conditions $(i),($ ii) and (iii) in Claim 2, which is absurd. Furthermore, in accordance with $(c)$ and $(d)$ in Claim 2, there is at most one $t \in \mathbb{R}$ such that $A_{t}^{-}$contains a special region.

It is clear that any region in $A_{t}^{-}$lies in a region of $A_{t^{\prime}}^{-}, t \leq t^{\prime}$. For any $t \in \mathbb{R}$, denote by $n_{t}$ the number of regions of $A_{t}^{-}$.

We say that $t \in \mathbb{R}$ is of the first kind if and only if there is an $\epsilon>0$ such that, for any $\left.t^{\prime} \in\right] t, t+\epsilon\left[, A_{t^{\prime}}^{-}\right.$contains a region disjoint By $A_{t}^{-}$. By Theorem 2.2 and the maximum principle, any region in $A_{t^{\prime}}^{-}$disjoint from $A_{t}^{-}$intersects $\partial(A)$, and so, it contains an open $\operatorname{arc} \gamma$ in $\partial(A)$ whose closure joins two points of $\left.A_{t^{\prime}}, t^{\prime} \in\right] t, t+\epsilon[$. Hence, the collection of points $P_{0} \in \gamma$ with $\left\langle X\left(P_{0}\right), v\right\rangle=\operatorname{Minimum}\{\langle X(P), v\rangle$ : $P \in \gamma\}$ is a non-empty and discrete set contained in $A_{t} \cap B$. Therefore, there are at most $\sharp(B)$ values of the first kind in $\mathbb{R}$, and if $t$ is of the first kind, then $\left.n_{t^{\prime}} \leq n_{t}+\sharp(B), t^{\prime} \in\right] t, t+\epsilon[$.

Define $I=\left\{t \in \mathbb{R}: A_{t}^{-}\right.$contains an embedded loop homotopic to $\left.\partial(A)\right\} \neq \emptyset$, and take $t_{I} \stackrel{\text { def }}{=} \operatorname{Infimum} I$. It is not hard to see that $I$ is open, and so $\left.I=\right] t_{I},+\infty[$, where maybe $t_{I}=-\infty$. From $(c)$ and $(d)$ in Claim 2, $A_{t^{\prime}}^{-}$does not contain any special region, $t^{\prime} \neq t_{I}$, and if $A_{t}^{-}$contains a special region then $t=t_{I}$.

Let $t_{0}$ be as in the hypothesis of the lemma.

Claim 3. For any $t \geq t_{0}, n_{t}$ and $\sharp\left(V_{t}\right)$ are finite numbers. Moreover, $n_{t} \leq n_{t_{0}}+\sharp(B)$ and $V_{t} \leq\left(n_{t_{0}}+\sharp(B)\right)^{2}$.

By Theorem 2.2, any region of $A_{t}^{-}$intersects $A_{t_{0}}^{-} \cup \partial(A), t \geq t_{0}$.

It is clear that at most $n_{t_{0}}$ regions of $A_{t}^{-}$intersect $A_{t_{0}}^{-}$. If a region of $A_{t}^{-}$meets $\partial(A)$, then it contains an open arc lying in $\partial(A)$ whose closure joins two points of $A_{t}$. Reasoning as above, it contains at least a point of $B$, and so there exist at most $\sharp(B)$ such regions. We deduce that $n_{t} \leq n_{t_{0}}+\sharp(B)<\infty$, and in accordance with (e) in Claim 2, $\sharp\left(V_{t}\right)<\infty$. As a matter of fact, $(a)$ and $(b)$ in Claim 2 and a combinatorial argument give $\sharp\left(V_{t}\right)<n_{t}^{2} \leq\left(n_{t_{0}}+\sharp(B)\right)^{2}$.

To complete the proof of the lemma, we reason by contradiction and suppose that $\bigcup_{t \geq t_{0}} V_{t}$ contains infinitely many points. By Claim 3, there exists an increasing sequence $\left\{t_{k}\right\}_{k \in \mathbb{N}} \subset\left[t_{0},+\infty\left[\right.\right.$ such that $V_{t_{k}} \neq \emptyset, k \in \mathbb{N}$. Observe that Claim 2 and Claim 3 imply that the sequences $\left\{\sharp\left(V_{t_{k}}\right)\right\}_{k \in \mathbb{N}}$ and $\left\{n_{t_{k}}\right\}_{k \in \mathbb{N}}$ are both bounded. Up to removing a finite set of values in the sequence, we can assume that $t_{I} \notin\left[t_{1}, t_{\infty}[\right.$, where $t_{\infty}=\operatorname{Supremum}\left\{t_{k}: k \in \mathbb{N}\right\}$. Furthermore, since the collection of values of the first kind is a finite set, we can also suppose that $\left[t_{1}, t_{\infty}\right.$ [ does not contain any such value. On the other hand, Theorem 2.2 implies that no region in $A_{t_{k+1}}^{-}$ is disjoint from $A_{t_{k}}^{-} \cup \partial(A)$, and thus, any region in $A_{t_{k+1}}^{-}$contains a region of 
$A_{t_{k}}^{-}$. Hence, $n_{t_{k+1}} \leq n_{t_{k}}, k \in \mathbb{N}$. Since $t_{I} \notin\left[t_{1}, t_{\infty}\right.$ [, any $v$-point in $V_{t_{k}}$ lies in the boundary of two distinct regions of $A_{t_{k}}^{-}$, and these two regions lie in the same region of $A_{t_{k+1}}^{-}$. So, in fact, $n_{t_{k}}>n_{t_{k+1}}, k \in \mathbb{N}$, which contradicts the fact that $\left\{n_{t_{k}}\right\}_{k \in \mathbb{N}}$ is a sequence of non-negative integer numbers.

Now we can prove

Theorem 3.1. Let $X: M \rightarrow \mathbb{R}^{3}$ be a properly immersed non-flat minimal surface of finite topology. Assume that $X$ has a finite plane $\Sigma$.

Then, the collection of points with normal vector orthogonal to $\Sigma$ is a finite set.

Proof. Since $M$ has finite topology, there is a compact subset $K \subset M$ such that $M-\stackrel{\circ}{K}$ is the union of a finite collection of once punctured closed disks. Moreover, since $\Sigma$ is a finite plane, we can choose $K$ in such a way that $(M-\stackrel{\circ}{K}) \cap X^{-1}\left(\mathbb{R}^{3}-\Sigma\right)$ contains finitely many connected components.

Since $K$ contains finitely many points with tangent plane parallel to $\Sigma$, it suffices to prove the theorem for properly immersed minimal annuli. The theorem follows from Lemma 3.1 .

3.1. Finite planes and finite total curvature. We are going to derive some basic consequences from Theorem 3.1.

Corollary 3.1. A properly immersed non-flat minimal surface of finite topology $X: M \rightarrow \mathbb{R}^{3}$ has finite total curvature if and only if $X$ has two finite non-parallel planes.

Proof. The geometry of minimal annular ends with finite total curvature is well known (see [21] and [12]). As a matter of fact, if $X$ has finite total curvature, any plane $\Sigma$ is a finite plane for $X$.

Let $X: M \rightarrow \mathbb{R}^{3}$ be a properly immersed minimal surface admitting two nonparallel finite planes, where $M$ has finite topology and compact boundary. Thanks to Theorem [2.3 $M$ is of finite conformal type. Since $X$ has two non-parallel finite planes, Theorem 3.1]implies that the Gauss map $N$ of $X$ has four exceptional values. In accordance with Picard's theorem, the conformal map $g=s \circ N$, where $s$ is the stereographic projection from the North Pole, extends conformally to the ends, and thus $X$ has finite total curvature.

As a consequence of this corollary, the only properly embedded simply connected minimal surface admitting two non-parallel finite planes is the plane. See Xavier 25] for a related result.

Corollary 3.2. Let $X: M \rightarrow \mathbb{R}^{3}$ be a properly immersed minimal surface of finite topology, $\partial(M)=\emptyset$. Assume there exist two non paralle! planes $\Sigma_{1}$ and $\Sigma_{2}$ such that $l_{j} \stackrel{\text { def }}{=} X(M) \cap \Sigma_{j}$ is a straight line, $j=1,2$.

Then, $X(M)$ is a plane.

Proof. Since $X$ is proper, the set $X^{-1}\left(l_{j}\right)$ consists of finitely many open simple arcs, and so $X^{-1}\left(\mathbb{R}^{3}-\Sigma_{j}\right)$ contains finitely many connected components, $j=1,2$; that is to say, $\Sigma_{1}$ and $\Sigma_{2}$ are finite planes for $X$. From Theorem [3.1, $X$ has finite total curvature.

\footnotetext{
${ }^{1}$ If $X$ has finite total curvature, we can substitute non-parallel for distinct, because any such surface of finite topology does not contain a pair of disjoint straight lines.
} 
In accordance with Schwarz's reflection principle, $X(M)$ is invariant under a $180^{\circ}$ rotation about the straight lines $l_{j}, j=1,2$. If $l_{1} \cap l_{2}=\emptyset$, the composition of these two rotations gives either a translation or a screw motion. However, no complete minimal surface with finite total curvature is invariant under such a rigid motion, and thus, $l_{1} \cap l_{2} \neq \emptyset$.

Let $A$ be an annular end of $M, A \cong] 0,1] \times \mathbb{S}^{1}$. As $X$ has finite total curvature, Osserman's theorem [21] implies that $A$ is conformally diffeomorphic to $\overline{\mathbb{D}}-\{0\}$, and the composition of the Gauss map of $X$ with the stereographic projection is a meromorphic map extending analytically to 0 .

Claim 1. $X(A)$ is a planar embedded end.

Assume for the moment that $X(A)$ includes a non-compact connected piece of a line $l_{j}, j \in\{1,2\}$. Up to removing a compact piece of $A$, we can suppose that $X(A)$ is invariant under a $180^{\circ}$ rotation about $l_{j}$. We denote by $\mathcal{R}_{j}$ the only antiholomorphic automorphism on $A \equiv \overline{\mathbb{D}}-\{0\}$ induced by this rotation (of course, we are understanding that $X \neq Y \circ p$, where $Y: N \rightarrow \mathbb{R}^{3}$ is a minimal immersion and $p: M \rightarrow N$ is a non-trivial covering map). It is clear that $\mathcal{R}_{j}$ extends conformally to 0 and $\mathcal{R}_{j}(0)=0$. Thus, up to a conformal transformation, we can suppose that $\mathcal{R}_{j}(z)=\bar{z}, z \in \overline{\mathbb{D}}$. Moreover, $X(M) \cap \Sigma_{j}=l_{j}$ implies that the fixed point set of $\mathcal{R}_{j}$ is $X^{-1}\left(l_{j}\right)$, and thus $X^{-1}\left(l_{j}\right) \cap A$ consists of two divergent curves.

Now we can prove the claim. Since $\Sigma_{1}$ and $\Sigma_{2}$ are not parallel, up to relabeling, we can assume that the limit normal vector of $A$ at the end is not orthogonal to $\Sigma_{2}$. So, $X(A) \cap \Sigma_{2} \neq \emptyset$, and it contains at least one divergent curve. As $X(A) \cap \Sigma_{2} \subset l_{2}$, $X(A)$ contains a non-compact piece of $l_{2}$. If the multiplicity of the annular end $X(A)$ is greater than 1 , then $A \cap X^{-1}\left(\Sigma_{2}\right)=X^{-1}\left(l_{2}\right)$ consists of at least four distinct divergent curves (see Theorem 2.1), which is absurd. Therefore, the multiplicity is 1 and $X(A)$ is an embedded end. Since $X(A)$ contains a straight line, it is planar.

Claim 2. Up to a compact set, $\left(l_{1} \cup l_{2}\right)$ is contained in $X(A)$.

Assume that $l_{1}-X(A)$ is not compact. Since $X(A)$ is an embedded planar end, we infer that $X(A) \cap l_{1}$ is compact, and so, up to removing a compact piece of $A$, $X(A) \cap \Sigma_{1}=\emptyset$. Hence, the normal vector at the end of $A$ is orthogonal to $\Sigma_{1}$, and thus it is not orthogonal to $\Sigma_{2}$. We deduce that $\Sigma_{2} \cap X(A)$ is not empty and consists of two divergent curves. Therefore, up to a compact set, $l_{2} \subset X(A)$, and the limit tangent plane at the end of $A$ contains $l_{2}$. But $l_{1} \cap l_{2} \neq \emptyset$ gives $\Sigma_{1} \cap l_{2} \neq \emptyset$. So $\Sigma_{1}$ is the limit tangent plane at the end of $A$, and it contains $l_{2}$. This fact contradicts $\Sigma_{1} \cap X(A)=\emptyset$, and proves that, up to a compact set, $l_{1} \subset X(A)$. In a similar way, and up to a compact set, $l_{2} \subset X(A)$, which proves the claim.

To finish the theorem, we distinguish two cases: $l_{1} \neq l_{2}$ and $l_{1}=l_{2}$.

If $l_{1} \neq l_{2}$, then the limit tangent plane at the end of $A$ is the only plane containing $l_{1} \cup l_{2}$. Since $A$ is an arbitrary annular end of $M$, all the planar ends of $M$ have the same limit tangent plane, and so $X(M)$ lies in a slab. Since $M$ is parabolic, we deduce that $X(M)$ is a plane. Assume now that $l_{1}=l_{2}$. In this case all the planar embedded ends contain the same straight line, namely $l \stackrel{\text { def }}{=} l_{1}=l_{2}$. Hence, $X^{-1}(P)$ contains as many points as $M$ has ends, $P \in X^{-1}(l)$. If $M$ has more than one end, this contradicts a well known consequence of the monotonicity formula for minimal surfaces (see [13] for details). So $M$ has only one embedded end, i.e., $X(M)$ is a plane. 
It is known that the only properly embedded minimal surface of finite total curvature meeting a plane in a straight line is the plane [3]. However, there exist complete immersed minimal surfaces with finite total curvature meeting a plane in a straight line. For instance, take $N=\mathbb{C}-\{0\}$ and

$$
X(z)=\operatorname{Re} \int_{i}^{z}\left(i\left(v^{-2}+v^{2}\right) d v,\left(v^{2}-v^{-2}\right) d v, 2 d v\right), \quad z \in N .
$$

The surface $X(N)$ has two ends, one of them embedded and of Riemann type, and the other one asymptotic to Meeks' Möbius strip. It is not hard to see that $X(N)$ meets the plane $\left\{x_{3}=0\right\}$ in the $x_{1}$-axis, which is a double straight line in $X(N)$ (see Figure 3).
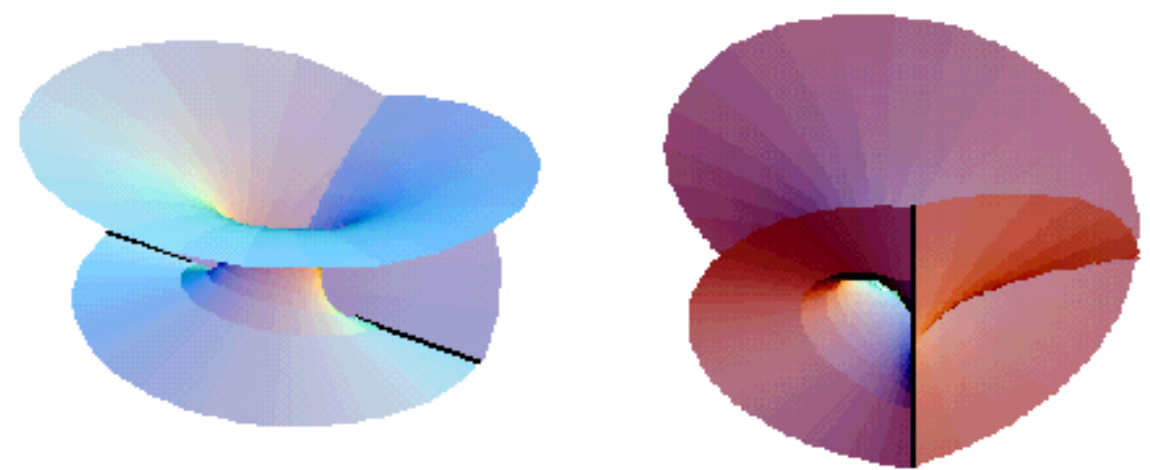

Figure 3. Two views of $X(N) \cap\left\{x_{3} \geq 0\right\}$.

\section{The ASYMPTOTIC BEHAVIOR OF PROPERLY IMMERSED MINIMAL SURFACES WITH FINITE TOPOLOGY}

This section is devoted to getting information about the set $\mathcal{B}(X(M))$, where $X: M \rightarrow \mathbb{R}^{3}$ is a properly immersed minimal surface with finite topology.

4.1. A characterization of minimal surfaces with finite total curvature. In this subsection we construct some collections of planar sectors in $\mathbb{R}^{3}$ (see Definition 4.1 below), that we have called stars of planar sectors, having the property of intersecting at infinity (i.e., outside any compact subset) any properly immersed minimal surface with finite topology and infinite total curvature. As a matter of fact, a complete minimal surface $X: M \rightarrow \mathbb{R}^{3}$ has finite total curvature if and only if there is a star of planar sectors eventually disjoint from $X(M)$.

The following notation is required (see Section 2). Let $\Pi$ be a plane in $\mathbb{R}^{3}$, and let $S^{\prime}$ be a sector in $\Pi$. If $B$ is an open Euclidean ball in $\mathbb{R}^{3}$, we refer to $S \stackrel{\text { def }}{=} \mathcal{E}\left(S^{\prime}-B\right)$ as a truncated planar sector in $\Pi$. We denote by $\partial(S)$ the boundary of $S$ as a topological surface. The base $\mathcal{B}(S)$ of $S$ is well defined and consists of a closed arc of the spherical geodesic $\mathbb{S}^{2} \cap \Pi_{0}$, where $\Pi_{0}$ is the only plane passing through the origin and parallel to $\Pi$. Up to translations and up to a compact set, $S$ is determined by $\mathcal{B}(S)$.

Let $L$ be a straight line, and let $P_{0}$ and $\Sigma$ be a point in $L$ and a plane orthogonal to $L$ and not containing $P_{0}$, respectively. Let $\mathcal{C}$ be a double cone in $\mathbb{R}^{3}$ with vertex 
$P_{0}$ and axis $L$, i.e., $\mathcal{C}=\left\{P_{0}+t v: t \in \mathbb{R}, v \in \gamma\right\}$, where $\gamma$ is a circle in $\Sigma$ centered at $\Sigma \cap L$. Let $\Omega(\mathcal{C})$ be the closure of the non-convex region of $\mathbb{R}^{3}-\mathcal{C}$. The plane orthogonal to the axis $L$ and passing through the vertex $P_{0}$ of $\mathcal{C}$ will be denoted by $\Sigma(\mathcal{C})$, and we also denote by $H_{i}(\mathcal{C}), i=1,2$, the two closed half spaces determined by $\Sigma(\mathcal{C})$. We define the angle of $\mathcal{C}$ as the angle made in $\Omega(\mathcal{C})$ by the two straight lines in $\mathcal{C} \cap \Pi$, where $\Pi$ is any plane satisfying $\Pi \perp \Sigma(\mathcal{C})$ and $L \subset \Pi$. We say that a double cone $\mathcal{C}^{\prime}$ is close enough to $\mathcal{C}$ if the vertex, the angle and the direction of the axis of $\mathcal{C}^{\prime}$ are close enough to the ones of $\mathcal{C}$.
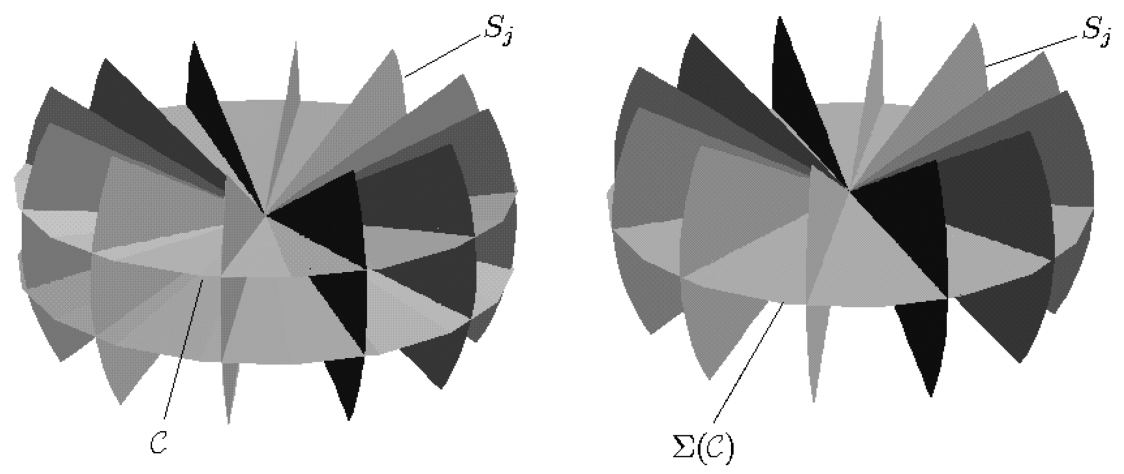

Figure $4 .|\mathcal{F}|, \mathcal{C}$ and $\Sigma(\mathcal{C})$.

Definition 4.1. Let $\mathcal{C}$ be a double cone, let $\mathcal{F}=\left\{S_{1}, \ldots, S_{r}\right\}$ be a finite family of pairwise eventually disjoint truncated planar sectors in $\mathbb{R}^{3}$, and let $|\mathcal{F}|=\bigcup_{j=1}^{r} S_{j}$. The family $\mathcal{F}$ is said to be a star of planar sectors associated to $\mathcal{C}$ if it satisfies:

(a) The planes $\Pi_{1}, \ldots, \Pi_{r}$ containing $S_{1}, \ldots, S_{r}$, respectively, are pairwise not parallel and the angles $a\left(S_{j}\right)$ lie in $] 0, \pi\left[, j=1, \ldots, r\right.$. Moreover, $\mathcal{E}(|\mathcal{F}|)=\mathbb{R}^{3}$ and $\mathcal{C}$ is transverse to $\mathcal{F}$, i.e., the end points of the spherical $\operatorname{arcs} \mathcal{B}\left(S_{j}\right), j=1, \ldots, r$, lie in different components of $\mathbb{S}^{2}-\mathcal{B}(\Omega(\mathcal{C}))$.

(b) There exists a Euclidean ball $B$ such that the following property is satisfied by any double cone $\mathcal{C}^{\prime}$ close enough to $\mathcal{C}$ :

If $S \subset H_{i}\left(\mathcal{C}^{\prime}\right)$ is a properly immersed connected minimal surface satisfying $S \cap(B \cup(|\mathcal{F}|))=\emptyset, \partial(S) \cap \Omega\left(\mathcal{C}^{\prime}\right) \neq \emptyset$ and $\partial(S) \cap \Omega\left(\mathcal{C}^{\prime}\right) \subset \Sigma\left(\mathcal{C}^{\prime}\right)$, then $S \subset \Sigma\left(\mathcal{C}^{\prime}\right)$, $i \in\{1,2\}$.

Note that if $\mathcal{F}$ is a star of planar sectors associated to $\mathcal{C}$, then $\mathcal{F}$ is also a star of planar sectors associated to any double cone $\mathcal{C}^{\prime}$ close enough to $\mathcal{C}$.

The next lemma yields a general method for constructing stars of planar sectors associated to double cones. We are specially interested in those with base disjoint from a given finite collection of spherical geodesics.

Lemma 4.1 (Existence of stars of planar sectors). Let $\Gamma=\left\{\gamma_{j}: j=1, \ldots, s\right\}$ be a (maybe empty) family of pairwise distinct spherical geodesics in $\mathbb{S}^{2}$. Define $|\Gamma|=\bigcup_{j=1}^{s} \gamma_{j}$ and $V=\left\{\gamma_{i} \cap \gamma_{h}: i \neq h\right\}$. Let $\Sigma_{0}$ be a plane in $\mathbb{R}^{3}$ passing through the origin such that $\gamma_{0}=\Sigma_{0} \cap \mathbb{S}^{2} \equiv \mathcal{B}\left(\Sigma_{0}\right)$ does not lie in $\Gamma$ and is disjoint from $V$.

Then, there exist a double cone $\mathcal{C}_{0}$ with vertex at the origin and a star of planar sectors $\mathcal{F}=\left\{S_{1}, \ldots, S_{r}\right\}$ associated to $\mathcal{C}_{0}$ satisfying $\Sigma\left(\mathcal{C}_{0}\right)=\Sigma_{0}$ and $\mathcal{B}(|\mathcal{F}|) \cap|\Gamma|=$ $\emptyset$. 
Proof. Let $v$ be a unit vector orthogonal to $\Sigma_{0}$, and let $t$ be the arc length parameter of $\gamma_{0}, \gamma_{0}(t):[0,2 \pi] \rightarrow \mathbb{S}^{2}, \gamma_{0}(0)=\gamma_{0}(2 \pi)$. Write $\gamma_{0}^{-1}(|\Gamma|)=\left\{t_{1}, \ldots, t_{k}\right\}$, where $t_{1}<t_{2}<\ldots<t_{k}$. Without loss of generality, we can assume that $t_{1}=0$, and so $t_{k}=2 \pi$.

Let $\gamma_{j(i)} \in \Gamma$ be the geodesic containing $\gamma_{0}\left(t_{i}\right)$, and let $v_{i}$ be the unit tangent vector of $\gamma_{j(i)}$ at $\gamma_{0}\left(t_{i}\right)$ satisfying $\left\langle v, v_{i}\right\rangle>0, i=1, \ldots, k$. Let $V$ denote a smooth field along $\gamma_{0}$ satisfying $\|V(t)\|=1,\left\langle V(t), \gamma_{0}(t)\right\rangle=0, t \in\left[0,2 \pi\left[, V\left(\gamma_{0}\left(t_{i}\right)\right)=v_{i}\right.\right.$, $i=1, \ldots, k-1$, and $\langle V(t), v\rangle>0, t \in\left[0,2 \pi\left[\right.\right.$. Since the vectors $\gamma_{0}^{\prime}(t)$ and $V(t)$ are linearly independent for $t \in\left[0,2 \pi\left[\right.\right.$, the smooth map $F:[0,2 \pi] \times \mathbb{R} \rightarrow \mathbb{S}^{2}$ given by

$$
F(t, s)=\exp _{\gamma_{0}(t)}(s V(t))
$$

satisfies $\operatorname{Jac}\left(F_{i}\right)(t, 0) \neq 0, t \in[0,2 \pi]$. Here, exp is the exponential map of the sphere $\mathbb{S}^{2}$.

Hence, it is not hard to find an $\epsilon>0$ small enough so that $\left.F\right|_{F^{-1}(U(\epsilon))}$ : $F^{-1}(U(\epsilon)) \rightarrow U(\epsilon)$ is one to one, where $U(\epsilon)$ is the spherical tubular neighborhood $\left\{p \in \mathbb{S}^{2}: \operatorname{dist}\left(p, \gamma_{0}\right) \leq \epsilon\right\}$.

For any $t \in[0,2 \pi]$, define $\alpha_{t}:[-\epsilon, \epsilon] \rightarrow U(\epsilon)$ by $\alpha_{t}(s)=F(t, s)$, and note that $\alpha_{t}$ is the piece of the geodesic $F(t, \cdot)$ contained in $U(\epsilon)$. In the following, each $\alpha_{t}$ will be identified with its image in $\mathbb{S}^{2}$.

Let $C$ be a truncated tetrahedral domain satisfying the hypothesis of Theorem 2.5, (b). The sets $\mathcal{B}(C)$ and $\mathcal{B}(\partial(C))$ are well defined, and $\mathcal{B}(\partial(C))=\mathcal{B}\left(F_{1}(C)\right) \cup$ $\mathcal{B}\left(F_{2}(C)\right) \cup \mathcal{B}\left(F^{+}(C)\right) \cup \mathcal{B}\left(F^{-}(C)\right)$. Furthermore, from Theorem 2.5 $(a), C$ can be chosen in such a way that the angles $\vartheta(C)$ and $\varrho(C)$ are greater that zero but as small as we want. Therefore, we can suppose

$$
\vartheta(C)>0 \text { and } \quad \text { diameter }(\mathcal{B}(C))<\epsilon
$$

where the diameter is computed in $\mathbb{S}^{2}$.

Let $\left.\epsilon^{\prime} \in\right]$ diameter $(\mathcal{B}(C)), \epsilon\left[\right.$, and denote by $\mathcal{U}\left(\epsilon^{\prime}\right)$ the family of double cones $\mathcal{C}$ with vertex in $\left\{x \in \mathbb{R}^{3}:\|x\|<1\right\}$ and satisfying:

$$
\left.\left\{p \in \mathbb{S}^{2}: \operatorname{dist}\left(p, \gamma_{0}\right) \leq \epsilon^{\prime}\right\} \subset \mathcal{B}(\stackrel{\circ}{\Omega(\mathcal{C}})\right) \subset \mathcal{B}(\Omega(\mathcal{C})) \subset \stackrel{\circ}{ }(\epsilon) .
$$

For any $\mathcal{C} \in \mathcal{U}\left(\epsilon^{\prime}\right)$, let $\gamma_{0}(\mathcal{C})$ denote the spherical geodesic $\mathcal{B}(\Sigma(\mathcal{C}))$.

Let $\left.\epsilon_{0} \in\right] \epsilon^{\prime}, \epsilon\left[\right.$, and let $\mathcal{C}_{0} \in \mathcal{U}\left(\epsilon^{\prime}\right)$ denote the double cone with vertex at the origin given by

$$
\mathcal{B}\left(\mathcal{C}_{0}\right)=\left\{p \in \mathbb{S}^{2}: \operatorname{dist}\left(p, \gamma_{0}\right)=\epsilon_{0}\right\} .
$$

We are going to show that $\mathcal{C}_{0}$ is the double cone whose existence is asserted by the lemma.

Indeed, if we choose $\left.\epsilon^{\prime} \in\right]$ diameter $(C), \epsilon$ [ close enough to $\epsilon$, it is not hard to find a small positive number $\kappa$ (which depends on $\epsilon^{\prime}$ and $C$ ) such that the following property is satisfied by any $\mathcal{C} \in \mathcal{U}\left(\epsilon^{\prime}\right)$ :

Given $t, t^{\prime} \in\left[0,2 \pi\left[,\left|t-t^{\prime}\right|<\kappa\right.\right.$, and $i \in\{1,2\}$, there is a rigid motion $R$ depending on $t, t^{\prime}, i$ and $\mathcal{C}$ such that the spherical domain $R(\mathcal{B}(C))$ is contained in $\mathcal{B}\left(H_{i}(\mathcal{C}) \stackrel{\circ}{\cap} \Omega(\mathcal{C})\right)$, the arcs $\alpha_{t} \cap \mathcal{B}\left(H_{i}(\mathcal{C})\right)$ nad $\alpha_{t^{\prime}} \cap \mathcal{B}\left(H_{i}(\mathcal{C})\right)$ do not meet $R\left(\mathcal{B}\left(F_{j}(C)\right)\right), j=1,2$, and both split $R(\mathcal{B}(C))$ into two domains. 


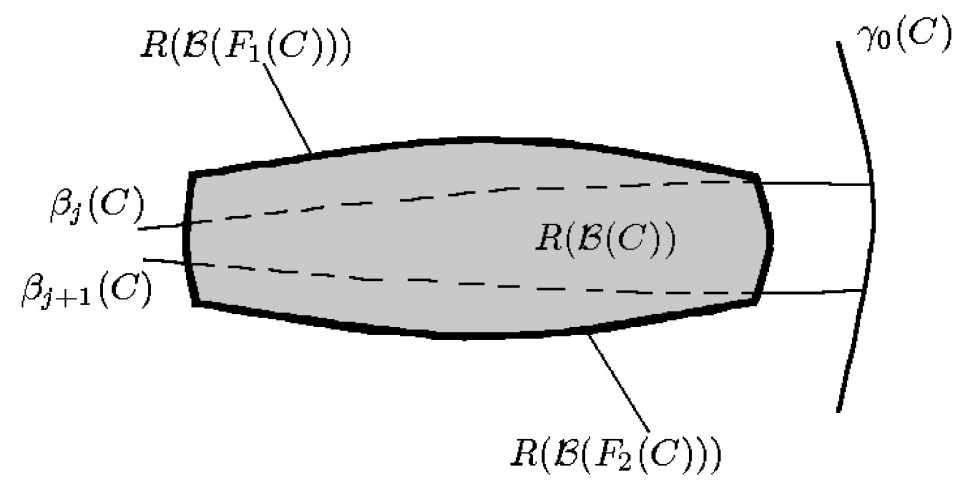

FiguRE 5 .

Let $\mathcal{P}=\left\{t_{0}, t_{1}^{\prime}, \ldots, t_{r+1}^{\prime}\right\} \subset[0,2 \pi]$ be a finite sequence of points satisfying $(i) 0=$ $t_{0}^{\prime}<t_{1}^{\prime}<t_{2}^{\prime}<\ldots<t_{r}^{\prime}<t_{r+1}^{\prime}=2 \pi ;(i i) t_{h}^{\prime} \notin\left\{t_{1}, \ldots, t_{k}\right\}$, for any $h \in\{1, \ldots, r\}$; and $($ iii $)\|\mathcal{P}\| \stackrel{\text { def }}{=}$ Minimum $\left\{t_{i+1}^{\prime}-t_{i}^{\prime}: i=0, \ldots, r\right\}<\kappa$. In the following, for simplicity, we denote by $\beta_{j}$ the geodesic $\alpha_{t_{j}^{\prime}}$. Write $\beta_{j}^{i}(\mathcal{C})=\beta_{j} \cap \mathcal{B}\left(H_{i}(\mathcal{C})\right)$, for any $j \in\{1, \ldots, r\}, i=1,2$ and $\mathcal{C} \in \mathcal{U}\left(\epsilon^{\prime}\right)$, and see Figure 5 for an explanation of this setting.

Since $\beta_{j}$ is disjoint from the great circle containing $\beta_{j+1}$ and vice versa, we can find a sequence of planar sectors $\left\{T_{1}, \ldots, T_{r+1}\right\}$, where $T_{r+1}=T_{1}$, such that $\mathcal{B}\left(T_{j}\right)=\beta_{j}$ and $l_{j} \stackrel{\text { def }}{=} T_{j} \cap T_{j+1}$ is a compact segment containing the origin as an interior point.

Thus, for any $j \in\{1, \ldots, r\}, i \in\{1,2\}$ and $\mathcal{C} \in \mathcal{U}\left(\epsilon^{\prime}\right)$, we can find a rigid motion $R_{j}^{i}(\mathcal{C})$ such that $(i)$ property (2) holds for $R=R_{j}^{i}(\mathcal{C})$ and $\left(t, t^{\prime}, i\right)=\left(t_{j}, t_{j+1}, i\right)$; (ii) the straight line $L_{j}$ containing $l_{j}$ is disjoint from $R_{j}^{i}(\mathcal{C})\left(F_{0}(C)\right)$; and $(i i i) L_{j}$ passes through the interior of $R_{j}^{i}(\mathcal{C})(C)$, meeting both faces $R_{j}^{i}(\mathcal{C})\left(F^{+}(C)\right)$ and $R_{j}^{i}(\mathcal{C})\left(F^{-}(C)\right)$ in a point.

Observe that, up to a homothetic expansion centered at the origin, the length of the segments $l_{j}$ can be as large as we want, and the same holds for the length of $l_{j}^{i}(\mathcal{C}) \stackrel{\text { def }}{=} l_{j} \cap H_{i}(\mathcal{C})$. Hence, and without loss of generality, we can suppose that the length of $L_{j} \cap R_{j}^{i}(\mathcal{C})(C)$ is less than the length of $l_{j}^{i}(\mathcal{C})$, for any $j \in\{1, \ldots, r\}$, $i \in\{1,2\}$ and $\mathcal{C} \in \mathcal{U}\left(\epsilon^{\prime}\right)$. Therefore, up to composing with a translation in the direction of $L_{j}$ which depends on $i$ and $\mathcal{C}$, we can assume that the closed segment $L_{j} \cap R_{j}^{i}(\mathcal{C})(C)$ lies in the interior of the segment $l_{j}^{i}(\mathcal{C})$, for any $j \in\{1, \ldots, r\}$, $i \in\{1,2\}$ and $\mathcal{C} \in \mathcal{U}\left(\epsilon^{\prime}\right)$.

If we label $A_{1}=R_{j}^{i}(\mathcal{C})(C)$ and $A_{2}=H_{i}(\mathcal{C}) \cap \Omega(\mathcal{C})$, these subsets satisfy the conditions under which (10) holds, and thus it is not hard to find a Euclidean ball $B$ such that $\bigcup_{j=1}^{r} l_{j} \subset B$ and

$$
R_{j}^{i}(\mathcal{C})(C)-B \subset H_{i}(\mathcal{C}) \cap \Omega(\mathcal{C}), \quad \partial\left(T_{j}\right) \cap \Omega(\mathcal{C}) \subset B,
$$

for any $j \in\{1, \ldots, r\}, i \in\{1,2\}$, and $\mathcal{C} \in \mathcal{U}\left(\epsilon^{\prime}\right)$. Then, we define $S_{j}=\mathcal{E}\left(T_{j}-B\right)$, $j=1, \ldots, r$, and $\mathcal{F}=\left\{S_{1}, \ldots, S_{r}\right\}$. It is clear that $\mathcal{F}$ is a family of pairwise disjoint planar sectors. We are going to see that $\mathcal{F}$ is a star of planar sectors associated to $\mathcal{C}_{0}$. Obviously, thanks to (2), (a) in Definition 4.1 holds. 


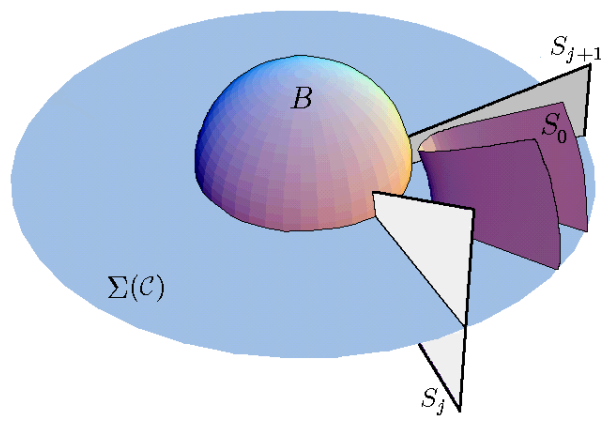

Figure 6 . The surface $S_{0}$.

Let $\mathcal{C} \in \mathcal{U}\left(\epsilon^{\prime}\right)$, and following the notation fixed in Definition 4.1 let $S$ be a properly immersed non-flat minimal surface in $H_{i}(\mathcal{C})$. Assume that $S \cap\left(B \cup\left(\bigcup_{j=1}^{r} S_{j}\right)\right)=$ $\emptyset, \emptyset \neq \partial(S) \cap \Omega(\mathcal{C}) \subset \Sigma(\mathcal{C})$ and $S \cap H_{i}(\mathcal{C}) \nsubseteq \Sigma(\mathcal{C})$. Then, taking into account the choice of $B$, there exists $j \in\{1, \ldots, r\}$ and a non-void connected component $S_{0}$ of $S \cap \Omega(\mathcal{C})$ satisfying $(i) S_{0}$ passes through the region in $H_{i}(\mathcal{C}) \cap \Omega(\mathcal{C})$ bounded by the truncated sectors $S_{j}$ and $S_{j+1}$ (see Figure 6), and so it must also pass through $R_{j}^{i}(\mathcal{C})(C)$ from $R_{j}^{i}(\mathcal{C})\left(F^{+}(C)\right)$ to $R_{j}^{i}(\mathcal{C})\left(F^{-}(C)\right) ;(i i) S_{0}$ does not touch $R_{j}^{i}(\mathcal{C})\left(\bigcup_{i=0}^{2} F_{i}(C)\right)$; and $(i i i) \partial\left(S_{0}\right)$ is disjoint from $R_{j}^{i}(\mathcal{C})(C)$. Hence, the surface $R_{j}^{i}(\mathcal{C})^{-1}\left(S_{0}\right) \cap C$ contradicts $(b)$ in Theorem 2.5 the desired contradiction. Therefore, $(b)$ in Definition 4.1 holds.

Since $|\mathcal{F}|=\bigcup_{j=1}^{r} \beta_{j}=\bigcup_{j=1}^{r} \alpha_{t_{j}^{\prime}}$, and the $\alpha_{t_{j}^{\prime}}$ are disjoint from $|\Gamma|, j=1, \ldots, r$, it follows that $|\mathcal{F}| \cap|\Gamma|=\emptyset$. This concludes the proof.

If $\Gamma=\emptyset$, the proof of the lemma is very simple. Indeed, we can take $V(t)$ as the only orthogonal, unitary and parallel field along $\gamma_{0}$ satisfying $\langle V(t), v\rangle>0$. Now, the map $F$ is injective in $\left[0,2 \pi[\times]-\frac{\pi}{2}, \frac{\pi}{2}\right.$ [, and so the truncated tetrahedral domain $C$ used in the proof (and satisfying the hypothesis of Theorem 2.5, $(b)$ ) can be chosen arbitrarily. In this case, take the sectors $T_{j}$ satisfying $(i)$ they are orthogonal to $\Sigma_{0}$ and symmetric with respect to this plane; $(i i) a\left(T_{j}\right)=a\left(T_{h}\right)$, $j \neq h$, where $\alpha \stackrel{\text { def }}{=} a\left(T_{j}\right), j=1 \ldots, r$, is greater than 2 diameter $(\mathcal{B}(C)) ;(i i i)$ the angle made by the planes containing $T_{j}$ and $T_{j+1}$ is small enough in terms of $\vartheta(C)$, in such a way that (2) holds (and so $r$ must be large enough); and (iv) $l_{j}=l_{h}$, $j \neq h$, where $l_{0} \stackrel{\text { def }}{=} l_{j}, j \in\{1, \ldots, r\}$, is a segment orthogonal to $\Sigma_{0}$, symmetric with respect to $\Sigma_{0}$, passing through the origin and of length greater than $2 \mathfrak{o}(C)$. Finally, choose any double cone $\mathcal{C}_{0}$ with vertex at the origin, axis orthogonal to $\Sigma_{0}$ and angle lying in $] 2 \operatorname{diameter}(\mathcal{B}(C)), \alpha\left[\right.$, and define $B$ and $S_{j}$ as in the proof of the lemma. The star of planar sectors $\mathcal{F} \stackrel{\text { def }}{=}\left\{S_{1}, \ldots, S_{r}\right\}$ associated to $\mathcal{C}_{0}$ so constructed is said to be simple.

Since the approach to the existence of barriers in [15] is constructive, it can be applied to numerical algorithms. Hence, the domains $C$ satisfying the hypothesis of Theorem [2.5, (b), and thus the star of sectors $\mathcal{F}$ in Lemma 4.1] can be determined by using a computer. 
Now we can prove

Theorem 4.1. Let $X: M \rightarrow \mathbb{R}^{3}$ be a properly immersed minimal surface of finite topology.

Then, $X$ has finite total curvature if and only if there exists a star of planar sectors $\mathcal{F}$ (associated to a double cone $\mathcal{C}$ ) such that $X(M)$ is eventually disjoint from $|\mathcal{F}|$.

Proof. Since $M$ has finite topology and compact boundary, it suffices to prove that the theorem is valid for annular ends $\left.\left.X: A \rightarrow \mathbb{R}^{3}, A \cong\right] 0,1\right] \times \mathbb{S}^{1}$. Furthermore, the theorem holds trivially when $X(M)$ lies in a plane. Hence, in the following, we will suppose that $M=A$ is an annulus and $X$ is not flat.

Suppose that $X(A) \cap|\mathcal{F}|$ is compact, where $\mathcal{F}=\left\{S_{1}, \ldots, S_{r}\right\}$ is a star of planar sectors associated to a double cone $\mathcal{C}$, and let us see that $\left.X\right|_{A}$ has finite total curvature.

From Definition 4.1, (b), the boundary of any connected component of $X(A)-$ $\Sigma(\mathcal{C})$ intersects $X(\partial(A)) \cup B \cup(X(A) \cap|\mathcal{F}|)$. As $X$ is proper, then $X(A)-\Sigma(\mathcal{C})$ has a finite number of non-compact connected components, and therefore, up to removing a compact subset of $A, \Sigma(\mathcal{C})$ is a finite plane for $X$. The same argument works for double cones close enough to $\mathcal{C}$, and thus $X$ admits infinitely many nonparallel finite planes. Lemma 3.1 and the Mo-Osserman theorem [22] (or Theorem 3.1) imply that $\left.X\right|_{A}$ has finite total curvature.

To finish the proof, assume now that $X$ has finite total curvature. From Theorem 2.1, the base $\mathcal{B}(X(M))$ is well defined and $\mathcal{B}(X(M))=\bigcup_{j=1}^{s} \gamma_{j}$, where the curves $\gamma_{j}$ are pairwise distinct spherical geodesics in $\mathbb{S}^{2}$. From Lemma 4.1, there exists a star of sectors $\mathcal{F}$ associated to a double cone $\mathcal{C}$ satisfying $\mathcal{B}(|\mathcal{F}|) \cap \mathcal{B}(X(M))=\emptyset$. It is not hard to see that $|\mathcal{F}| \cap X(M)$ is compact, which completes the proof.

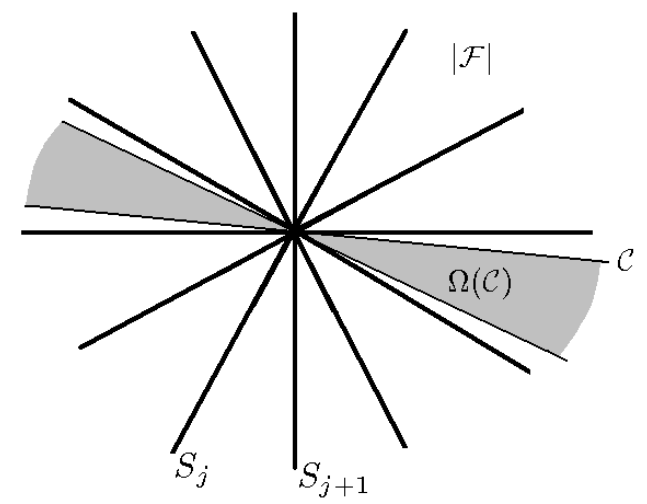

Figure 7. A two-dimensional view of $|\mathcal{F}|$ and $\mathcal{C}$.

As a consequence, we have

Corollary 4.1 (Cone lemma, Hoffman and Meeks 10]). There exists a shallow enough double cone $\mathcal{C}$ such that the following statement holds:

If $X: A \rightarrow \mathbb{R}^{3}$ is a properly immersed minimal annulus, $\left.\left.A \cong\right] 0,1\right] \times \mathbb{S}^{1}$, and $X(A)$ is eventually disjoint from $\mathcal{C}$, then $X$ has finite total curvature. 
Proof. We use Lemma 4.1 to construct a simple star of planar sectors $\mathcal{F}$ associated to a double cone $\mathcal{C}_{0}$. Obviously, there exists a shallow enough double cone $\mathcal{C}$ such that $|\mathcal{F}| \cap \Omega(\mathcal{C})$ is compact (see Figure[7). Assume that $X(A) \cap \mathcal{C}$ is compact. By the convex hull property (see [10] or Theorem 2.4), the closure of $X(A) \cap\left(\mathbb{R}^{3}-\Omega(\mathcal{C})\right.$ ) is compact, and so $X(A) \cap|\mathcal{F}|$ is compact. In accordance with Theorem $4.1, X$ has finite total curvature.

4.2. Minimal surfaces with flat ends. In this subsection we obtain a characterization of minimal surfaces with planar ends.

We start with the following lemma, which contains quite a lot of information about the geometry of properly immersed minimal discs with boundary in a wedge of $\mathbb{R}^{3}$.

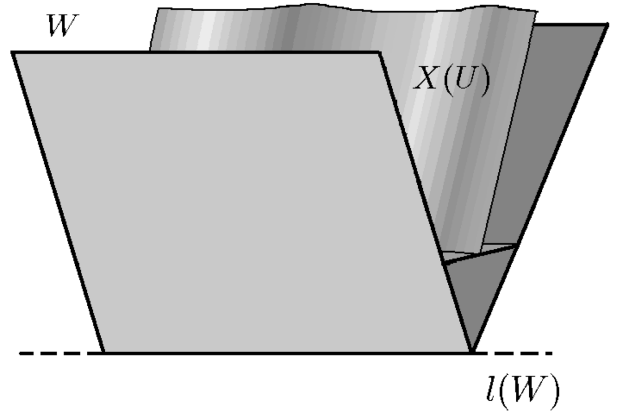

Figure $8 . W$ and $X(U)$.

Lemma 4.2. Let $W \subset\left\{x_{3} \geq 0\right\}$ be a wedge of $\mathbb{R}^{3}$ satisfying $a(W)<\pi, l(W)$ is the $x_{2}$-axis, and $\Pi(W)=\left\{x_{1}=0\right\}$. Let $X \equiv\left(X_{1}, X_{2}, X_{3}\right): U \rightarrow \mathbb{R}^{3}$ be a proper conformal minimal immersion, where $U$ is homeomorphic to a closed disc minus a boundary point. Assume that $X(U) \subset W$ and $X_{3}(\partial(U))$ is bounded (see Figure 8).

Then, $X(U)$ lies in a slab $\mathcal{S}$. Moreover, if $X_{3}(U)$ is not bounded, then:

(a) There exists $T>0$ such that $X_{3}^{-1}([T,+\infty[)$ is a simply connected graph over any plane $\Lambda$ parallel to $\mathcal{S}$.

(b) If $\left\{P_{n}\right\}_{n \in \mathbb{N}} \subset U$ and $\lim _{n \rightarrow \infty} X_{3}\left(P_{n}\right)=+\infty$, then $\lim _{n \rightarrow \infty} N\left(P_{n}\right)=v_{0}$, where $N$ is the Gauss map of $X$ and $v_{0}$ is orthogonal to $\Lambda$.

Proof. There is $t_{0}>0$ large enough so that $\left\{x_{3} \geq t_{0}\right\} \cap X(\partial(U))=\emptyset$.

In the following, and up to the translation $x \rightarrow x-\left(0,0, t_{0}\right)$, we suppose that $X(U) \subset\left\{x_{3} \geq-t_{0}\right\}$ and $X_{3}(\partial(U)) \subset\left\{x_{3}<0\right\}$. For simplicity, the wedge $\left(0,0,-t_{0}\right)+W$ will be also denoted by $W$.

If $X_{3}^{-1}(0)=\emptyset$, then $X(U)$ lies in the solid horizontal cylinder $W \cap\left\{x_{3}<0\right\}$, and the lemma holds.

In what follows, we assume that $X_{3}^{-1}(0) \neq \emptyset$. By Theorem 2.2, we deduce that, in fact, $X_{3}^{-1}(t) \neq \emptyset, t \geq 0$.

Claim 1. For any $\left.\left.t \geq 0, \Omega_{t} \stackrel{\text { def }}{=} X_{3}^{-1}(]-\infty, t\right]\right)$ and $U_{t} \stackrel{\text { def }}{=} X_{3}^{-1}([t,+\infty[)$ are simply connected. In particular, $\partial\left(U_{t}\right)=X_{3}^{-1}(t)$ is a simple arc, $t \geq 0$.

Let $\Omega$ be a connected component of $U_{t}, t \geq 0$. Thanks to Theorem 2.4, $X_{2}(\partial(\Omega))$ is not bounded either from above or from below. On the other hand, Theorem 2.2 
implies that any connected component of $\Omega_{t}$ contains $\partial(U)$, and so $\Omega_{t}$ is connected, $t \geq 0$. Therefore, any connected component of $U_{t}$ is bounded by only one simple arc whose image under $X_{2}$ is the whole real line, $t \geq 0$. Since $X$ is proper, we deduce that $U_{t}$ contains a finite number of connected components, and so $\Omega_{t}$ is simply connected and bounded by a finite set of pairwise disjoint, divergent and regular simple arcs, and this for any $t \geq 0$. In particular, no point of $U_{t}$ has vertical normal vector, $t \geq 0$. Assume that $U_{t}$ is not connected; that is to say, $U_{t}$ contains at least two connected components, $t \geq 0$. Then we can find a closed domain $D_{t} \subset \bar{\Omega}_{t}$ homeomorphic to a closed disc minus one boundary point and such that $\partial\left(D_{t}\right) \cap \partial(U)=\emptyset$ and $\partial\left(D_{t}\right)-\partial\left(\Omega_{t}\right)$ is compact (see Figure 9). From [6], $\overline{D_{t}}$ is parabolic, and so there exists a homeomorphism $F: D_{t} \rightarrow \overline{\mathbb{D}}-\{1\}$ which is holomorphic on $\stackrel{\circ}{D}_{t}$ (see [8]). Since $X_{3} \circ F^{-1}$ is bounded and continuous on $\overline{\mathbb{D}}-\{1\}$, harmonic in $\mathbb{D}$, and constant and equal to $t$ in a neighborhood of 1 in $\partial(\overline{\mathbb{D}})$, then $X_{3} \circ F^{-1}$ extends continously to $\overline{\mathbb{D}}$ taking the value $t$ on 1 . Therefore, $X\left(\overline{D_{t}}\right)$ is asymptotic at infinity to the plane $\left\{x_{3}=t\right\}$.

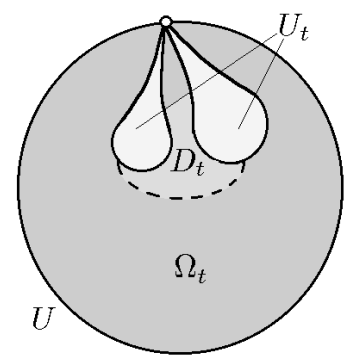

Figure $9 . \Omega_{t}, U_{t}$ and $D_{t}$.

Let $t^{\prime}>t$. From Theorem 2.2, $U_{t^{\prime}}$ meets any connected component of $U_{t}$, and so, reasoning as above, we can find a closed domain $D_{t^{\prime}} \subset \bar{\Omega}_{t^{\prime}}$ homeomorphic to $\overline{\mathbb{D}}-\{1\}$ and such that $D_{t^{\prime}} \subset D_{t}, \partial\left(D_{t^{\prime}}\right) \cap \partial(U)=\emptyset$ and $\partial\left(D_{t^{\prime}}\right)-\partial\left(\Omega_{t^{\prime}}\right)$ is compact. As above, $X\left(\overline{D_{t^{\prime}}}\right)$ must be asymptotic at infinity to the plane $\left\{x_{3}=t^{\prime}\right\}$, which gets a contradiction $\left(t \neq t^{\prime} ! !\right)$ and proves the claim.

Denote by $X_{3}^{*}$ the harmonic conjugate of $X_{3}$, and define $h: U_{0} \rightarrow\{z \in \mathbb{C}:$ $\operatorname{Re}(z) \geq 0\}, h(P) \stackrel{\text { def }}{=} X_{3}(P)+i X_{3}^{*}(P)$. As a consequence of Claim 1 , the third coordinate function $X_{3}$ has no singular point in $U_{0}$, and thus $h$ is a holomorphic and injective function. As $U_{0}$ is parabolic (see [6]), $h\left(U_{0}\right)=\{z \in \mathbb{C}: \operatorname{Re}(z) \geq 0\}$.

In what follows, we define $U_{t} \equiv h\left(U_{t}\right)=\{z \in \mathbb{C}: \operatorname{Re}(z) \geq t\}, t \geq 0$, and if $t \leq 0$, we define $U_{t}=\{z \in \mathbb{C}: \operatorname{Re}(z) \geq t\}$ too.

Note that the third holomorphic 1-form in the Weierstrass representation of $X\left(U_{0}\right)$ is given by $\Phi_{3}(z)=d z, z \in U_{0}$, and the Gauss map $g$ of $X$ is a zero-free holomorphic function in $U_{0}$. Take $t>0$, and let $\mathcal{Q}_{t}$ be the collection of planes whose intersection with $W$ lies in the open slab $\left\{0<x_{3}<t\right\}$. Take $\Upsilon \in \mathcal{Q}_{t}$, and let $\Upsilon^{+}$ be the connected component of $\mathbb{R}^{3}-\Upsilon$ containing $X\left(U_{t}\right)$. Reasoning as in Claim 1 , the family of planes which are parallel to $\Upsilon$ induces a foliation of $X^{-1}\left(\Upsilon^{+}\right)$by simple arcs, and so $g\left(U_{t}\right)$ omits the two normal vectors of $\Upsilon$. Hence, $g$ omits on $U_{t}$ the collection $V_{t}$ of normal vectors of planes $\Upsilon \in \mathcal{Q}_{t}$. It is clear that $V_{t}$ is an open subset of $\mathbb{R} \cup\{\infty\} \subset \overline{\mathbb{C}}$ containing 0 and $\infty$, and invariant under the antipodal map. 
In particular, $g$ omits on $U_{t}, t>0$, more than three points of $\overline{\mathbb{C}}$. Note that $V_{t} \subset V_{t^{\prime}}$, $t \leq t^{\prime}$, and define $V=\bigcup_{t>0} V_{t}$. It is clear that $V$ is the open arc in $\mathbb{R} \cup\{\infty\}$ defined by the normal vectors of the planes meeting $W$ in $l(W)$.

In the following, we set $A=\bigcap_{t \geq 0} g\left(U_{t}\right)$ and

$$
A_{0}=\left\{z \in \overline{\mathbb{C}}: \liminf _{t \rightarrow \infty} \chi\left(z, g\left(U_{t}\right)\right)=0\right\},
$$

where $\chi$ is the standard metric in the Riemann sphere. Note that $V \cap A=\emptyset$ and $A \subset A_{0}$.

Claim 2. $A_{0} \cap V=\emptyset$. In particular, if $t>0$ is large enough, $X\left(U_{t}\right)$ is a minimal graph.

A point $w \in \overline{\mathbb{C}}$ belongs to $A_{0}$ if and only if there exist a sequence $\left\{z_{n}\right\}_{n \in \mathbb{N}}$ in $U_{0}$ satisfying $\lim _{n \rightarrow \infty} \operatorname{Re}\left(z_{n}\right)=+\infty$ and $\lim _{n \rightarrow \infty} g\left(z_{n}\right)=w$. Let $w \in A_{0}$, and take $\left\{z_{n}\right\}_{n \in \mathbb{N}} \subset U_{0}$ as such a sequence. Up to a rigid motion, we can suppose that $w \neq 0, \infty$ (in this case, if necessary, choose a new $W$ containing $X(U)$ but keeping $l(W)$ and $\Pi(W)$ invariant $)$.

Let $S_{n}$ denote the surface defined by the homothetical shrinking

$$
\frac{1}{X_{3}\left(z_{n}\right)} \cdot\left(-X\left(z_{n}\right)+X\left(U_{0}\right)\right) \text {. }
$$

To be more precise, define the Weierstrass data $g_{n}(u)=g\left(\operatorname{Re}\left(z_{n}\right) u+z_{n}\right), \Phi_{3}(u)=$ $d u$ on $U_{-1}$, and consider the associated minimal immersion

$$
X^{n}(u)=\int_{0}^{u}\left(\frac{1}{2}\left(\frac{1}{g_{n}(v)}-g_{n}(v)\right), \frac{i}{2}\left(\frac{1}{g_{n}(v)}+g_{n}(v)\right), 1\right) d v .
$$

As $X_{3}(z)=\operatorname{Re}(z), z \in U_{0}$, it is clear that $S_{n}=X^{n}\left(U_{-1}\right)$.

Since the family $\left\{\left.\left(g_{n}\right)\right|_{U_{-1+\epsilon}}: n \in \mathbb{N}\right\}$ omits three values of $\overline{\mathbb{C}}$, and this holds for any $\epsilon>0$, then $\left\{g_{n}: n \in \mathbb{N}\right\}$ is normal on $U_{-1}^{\circ}$. So, up to taking a subsequence, $\left\{g_{n}\right\}_{n \in \mathbb{N}}$ converges on compact subsets of $U_{-1}$, as $n \rightarrow \infty$, to a meromorphic function $G: U_{-1}^{\circ} \rightarrow \overline{\mathbb{C}}$. Therefore, the sequence $\left\{X^{n}\right\}_{n \in \mathbb{N}}$ converges on compact subsets of $\stackrel{\circ}{U}_{-1}$ to $Y: \stackrel{\circ}{U}_{-1} \rightarrow \mathbb{R}^{3}$, where

$$
Y(u)=\int_{0}^{u}\left(\frac{1}{2}\left(\frac{1}{G(v)}-G(v)\right), \frac{i}{2}\left(\frac{1}{G(v)}+G(v)\right), 1\right) d v .
$$

Assume that $G$ is constant, i.e., $G \equiv w$ (remember that $G(0)=w$ ). In this case, $Y\left(\stackrel{\circ}{U}_{-1}\right)$ is a half plane whose normal vector projects stereographically on $w$. Since $X\left(U_{0}\right) \subset W$ and $X_{3}(z)=\operatorname{Re}(z)$, the sequence $\left\{\frac{1}{\operatorname{Re}\left(z_{n}\right)} \cdot X_{1}\left(z_{n}\right)\right\}_{n \in \mathbb{N}}$ is bounded, and so there exists a wedge $W^{\prime}$ such that $W \subset W^{\prime}, a\left(W^{\prime}\right)=a(W)$ and $\bigcup_{n \in \mathbb{N}} S_{n} \subset W^{\prime}$. This implies that $Y\left(\stackrel{\circ}{U}_{-1}\right) \subset W^{\prime}$, and so $w \in \mathbb{R}-V$.

Suppose now that $G$ is not constant. Hence, $G$ is holomorphic and $G(0)=w$. In particular, $G$ is open. Let $D(z, R) \subset \mathbb{C}$ be the disc of radius $R$ centered at $z$, and take $R \in] 0,1[$ and $\epsilon>0$ such that $D(w, 2 \epsilon) \subset G(D(0, R))-G(\partial(D(0, R)))$. Since $\left\{g_{n}\right\}_{n \in \mathbb{N}} \rightarrow G$ uniformly on $\overline{D(0, R)}$, there is $N \in \mathbb{N}$ such that $D(w, \epsilon) \cap$ $g_{n}(\partial(D(0, R)))=\emptyset, n \geq N$. By the Hurwitz theorem, $D(w, \epsilon) \subset g_{n}(D(0, R))$ for $n$ large enough, and thus $D(w, \epsilon) \subset \bigcap_{n \in \mathbb{N}} g\left(U_{(1-R) \operatorname{Re}\left(z_{n}\right)}\right)=\bigcap_{t \geq 0} g\left(U_{t}\right)$. This means that $w \in \stackrel{\circ}{A}$. 
Summarizing, we have proved that

$$
A \subset A_{0} \subset(\mathbb{R}-V) \cup \stackrel{\circ}{A} .
$$

Taking into account that $A_{0}$ is a closed subset of $\overline{\mathbb{C}}$, we conclude that $\operatorname{Fr}\left(A_{0}\right) \stackrel{\text { def }}{=}$ $A_{0}-\stackrel{\circ}{A_{0}} \subset A_{0}-\stackrel{\circ}{A} \subset \mathbb{R}-V$. Hence, any connected component of $A_{0}-(\mathbb{R}-V)$ is open and closed in $\overline{\mathbb{C}}-(\mathbb{R}-V)$, and so, either $A_{0} \subset(\mathbb{R}-V)$ or $\overline{\mathbb{C}}-(\mathbb{R}-V) \subset A_{0}$. If the second possibility holds, then $V \subset A_{0}$, that is to say, $V \subset \stackrel{\circ}{A}$, which is obviously absurd. This proves that $A_{0} \subset \mathbb{R}-V$.

Finally, since $g\left(U_{t}\right)$ is connected and lies in an arbitrarily small neighborhood of $\mathbb{R}-V, t$ large enough, the image under the Gauss map of $X\left(U_{t}\right)$ lies in a hemisphere of $\mathbb{S}^{2}$. It is not hard to see that $X\left(U_{t}\right)$ is a graph over, for instance, the plane $\left\{x_{1}=0\right\}, t$ large enough. This proves the claim.

Let $l_{1}$ and $l_{2}$ be the two parallel boundary lines of $\left\{x_{3}=0\right\} \cap W$, and let $W_{j}$ denote the smallest wedge in $\left\{x_{3} \geq 0\right\}$ satisfying $l\left(W_{j}\right)=l_{j}$ and $X\left(U_{0}\right) \subset W_{j}$, $j=1,2$. Since $X\left(\partial\left(U_{0}\right)\right) \subset\left\{x_{3}=0\right\}$, one of the two half planes in $\partial\left(W_{j}\right)$ lies in $\left\{x_{3}=0\right\}, j=1,2$, and so $W_{1} \cap W_{2}$ is the truncated wedge bounded by the two half planes $\Pi_{1}^{\prime} \subset \partial\left(W_{1}\right), \Pi_{2}^{\prime} \subset \partial\left(W_{2}\right)$ that are not contained in $\left\{x_{3}=0\right\}$, and the strip $\left\{x_{3}=0\right\} \cap W$. Let $W_{0}$ denote the only wedge satisfying $W_{0} \cap\left\{x_{3} \geq 0\right\}=W_{1} \cap W_{2}$, and observe that $X\left(U_{0}\right) \subset W_{0}$. Let $\Pi_{j}$ denote the plane containing $\Pi_{j}^{\prime}, j=1,2$.

Claim 3. $\lim \inf _{t \rightarrow+\infty} \frac{d\left(X\left(\partial\left(U_{t}\right)\right), \Pi_{j}\right)}{t}=0, j=1,2$, where $d$ means Euclidean distance.

Assume there is $j \in\{1,2\}$ such that $\liminf _{t \rightarrow+\infty} \frac{d\left(X\left(\partial\left(U_{t}\right)\right), \Pi_{j}\right)}{t}=C>0$. Then it is not hard to find a wedge $W_{j}^{\prime} \subset\left\{x_{3} \geq 0\right\}$ such that $l\left(W_{j}^{\prime}\right)=l_{j}$, a face of $\partial\left(W_{j}^{\prime}\right)$ lies in $\left\{x_{3}=0\right\}, a\left(W_{j}^{\prime}\right)<a\left(W_{j}\right)$ (and thus, $\left.W_{j}^{\prime} \subset W_{j}\right)$, and $X\left(U_{t^{\prime}}\right) \subset W_{j}^{\prime}$ for $t^{\prime}>0$ large enough.

Let us show that $X\left(U_{0}\right) \subset W_{j}^{\prime}$. Otherwise, $X\left(U_{0}\right)-W_{j}^{\prime}$ would be a properly immersed minimal surface in the solid cylinder $W \cap\left\{0 \leq x_{3} \leq t^{\prime}\right\}$ and with planar boundary lying in $\partial\left(W_{j}^{\prime}\right)-\left\{x_{3}=0\right\}$. Using Theorem 2.2, we would deduce that $X\left(U_{0}\right)-W_{j}^{\prime}$ is a collection of planar domains, which is absurd. Therefore, $W_{j}^{\prime}$ contains $X\left(U_{0}\right)$ and is smaller that $W_{j}$, which contradicts the choice of $W_{j}$ and proves the claim.

Claim 4. For any $a \in \mathbb{R}, \lim \sup _{t \rightarrow+\infty} \frac{\left|X_{2}(t+a i)\right|}{t}=0$.

Note that

$$
\frac{d X_{2}(t+a i)}{d t}=-\frac{1}{2} \operatorname{Im}\left(g(t+a i)+\frac{1}{g(t+a i)}\right),
$$

and so Claim 2 gives

$$
\lim _{t \rightarrow+\infty} \frac{d X_{2}(t+a i)}{d t}=0
$$

The claim follows easily.

Now we can prove the lemma. Define $U_{0}^{+}=\left\{z \in U_{0}: \operatorname{Im}(z) \geq 0\right\}$ and $U_{0}^{-}=$ $\left\{z \in U_{0}: \operatorname{Im}(z) \leq 0\right\}$. Since $X$ is proper and $X\left(\partial\left(U_{0}\right)\right) \equiv X(\{i a: a \in \mathbb{R}\})$ lies in the strip $\left\{x_{3}=0\right\} \cap W_{0}$, we have $\lim _{|t| \rightarrow+\infty}\left|X_{2}(i t)\right|=+\infty$. On the other hand, as we mentioned at the beginning of the proof of the lemma, $X_{2}\left(\partial\left(U_{0}\right)\right)$ is not bounded from above or from below. Hence, and without loss of generality, we can assume that $\lim _{t \rightarrow+\infty} X_{2}(i t)=+\infty$ and $\lim _{t \rightarrow-\infty} X_{2}(i t)=-\infty$. From Claim 4, the boundary 
of $X\left(U_{0}^{+}\right)$lies in a cone, and the same holds for $X\left(U_{0}^{-}\right)$. Thus, Theorem 2.4 gives that $X\left(U_{0}^{+}\right)$lies in the convex hull of its boundary, and the same occurs for $X\left(U_{0}^{-}\right)$. Therefore, $X\left(U_{0}\right) \subset W_{0}^{\prime} \subset W_{0}$, where $W_{0}^{\prime}=\mathcal{E}\left(\left(\left\{x_{3}=0\right\} \cap W_{0}\right) \cup X([0,+\infty[))\right.$. So, taking into account the definition of $W_{0}$ and Claim 3, it is not hard to deduce that

$$
\liminf _{t \rightarrow+\infty} \frac{d\left(X(t), \Pi_{j}\right)}{t}=0, \quad j=1,2 .
$$

Hence, letting $v_{j}$ be the normal vector of $\Pi_{j}$ pointing to $W_{0}$, the harmonic function $f_{j}: U_{0} \rightarrow \mathbb{R}, f_{j}(z) \stackrel{\text { def }}{=}\left\langle X(z), v_{j}\right\rangle$ satisfies $(i)$ it is bounded from below; (ii) $\left|f_{j}\right|$ is bounded on $\partial\left(U_{0}\right)$; and (iii) $\liminf _{t \rightarrow+\infty} \frac{f_{j}(t)}{t}=0, j=1,2$. By Jorgensen's theorem (see [2], pp.164, 284), $f_{j}$ is bounded, $j=1,2$, and so $\Pi_{1} \| \Pi_{2}, v_{1}=-v_{2}$, and $W_{0}$ is a slab. Therefore, taking into account Theorem 2.2 once again, it is not hard to check that $X(U)$ lies in a wider slab $\mathcal{S}$ containing $W_{0}$, which proves the first part of the lemma and $(a)$.

To see $(b)$, let $\left\{z_{n}\right\}_{n \in \mathbb{N}}$ be a sequence in $U_{0}$ satisfying $\left\{\operatorname{Re}\left(z_{n}\right)\right\} \rightarrow+\infty$, and define $Y^{n}(v)=X\left(v+z_{n}\right)-X\left(z_{n}\right), z \in U_{-\operatorname{Re}\left(z_{n}\right)}, n \in \mathbb{N}$. Using Claim 2, and reasoning as in the proof of this claim, the sequence $\left\{Y^{n}\right\}_{n \in \mathbb{N}}$ converges in a natural way to a complete minimal surface $Y: \mathbb{C} \rightarrow \mathbb{R}^{3}$ contained in $\mathcal{S}$, and whose Gauss map omits $\overline{\mathbb{C}}-(\mathbb{R}-V)$. By the Picard theorem, the Gauss map of $Y$ is constant and $Y(\mathbb{C})$ is a plane parallel to $\mathcal{S}$. So, $\left\{N\left(z_{n}\right)\right\} \rightarrow v_{0}$, where $v_{0}$ is a normal vector of this plane, which proves $(b)$ and the lemma.

Corollary 4.2. Let $X: U \rightarrow \mathbb{R}^{3}$ and $W$ be as in Lemma 4.2, and assume that $X_{3}^{-1}(t) \neq \emptyset$, for any $t>0$. Let $\Pi$ be a plane which is not parallel to $l(W)$.

Then, $X^{-1}\left(\Pi \cap\left\{x_{3} \geq 0\right\}\right)$ contains only one divergent simple arc.

The following lemma deals with a non-existence theorem for properly immersed minimal surfaces in solid right cylinders over a quadrilateral. The proof is an easy consequence of some existence results of minimal graphs by Jenkins and Serrin [11] and the maximum principle.

Let $D$ be a convex quadrilateral in $\left\{x_{3}=0\right\}$ with edges $A_{1}, C_{1}, A_{2}$ and $C_{2}$, where

$$
A_{1} \cap A_{2}=C_{1} \cap C_{2}=\emptyset .
$$

Let $V$ denote the set of vertices of $\partial(D)$, that is, the endpoints of the four edges of $D$. Let $\mathfrak{p}_{3}: \mathbb{R}^{3} \rightarrow\left\{x_{3}=0\right\}$ denote the orthogonal projection, and set $\mathcal{D}=\mathfrak{p}_{3}^{-1}(D)$, $\mathcal{A}_{i}=\mathfrak{p}_{3}^{-1}\left(A_{i}\right)$ and $\mathcal{C}_{i}=\mathfrak{p}_{3}^{-1}\left(C_{i}\right), i=1,2$. Let $\left|A_{i}\right|$ and $\left|C_{i}\right|$ be the lengths of $A_{i}$ and $C_{i}$, respectively, $i=1,2$.

Lemma 4.3. If $\left|A_{1}\right|+\left|A_{2}\right|<\left|C_{1}\right|+\left|C_{2}\right|$, then there are no properly immersed non-flat minimal surfaces $S$ in $\mathbb{R}^{3}$ such that $S \subset \mathcal{D} \cap\left\{x_{3} \geq 0\right\}$ and $\partial(S) \subset\left(\mathcal{A}_{1} \cup\right.$ $\left.\mathcal{A}_{2}\right)-\mathfrak{p}^{-1}(V)$.

Proof. By results of Jenkins and Serrin [11], there exists a unique properly immersed minimal surface $G \subset \mathcal{D} \cap\left\{x_{3} \leq 0\right\}$ such that: $\left.(i) \mathfrak{p}_{3}\right|_{G-\left(\mathcal{A}_{1} \cup \mathcal{A}_{2}\right)}: G-\left(\mathcal{A}_{1} \cup \mathcal{A}_{2}\right) \rightarrow$ $D-\left(A_{1} \cup A_{2}\right)$ is one-to-one and $\partial(G)=\left(C_{1} \cup C_{2}\right) \cup\left(\mathfrak{p}_{3}^{-1}(V) \cap\left\{x_{3} \leq 0\right\}\right)$; and (ii) if $\left\{P_{n}\right\}_{n \in \mathbb{N}} \in G$ and $\left\{\mathfrak{p}_{3}\left(P_{n}\right)\right\} \rightarrow P \in\left(A_{1} \cup A_{2}\right)-V$, then $\left\{y\left(P_{n}\right)\right\} \rightarrow-\infty$.

Reasoning by contradiction, assume there exists $S$ satisfying the above conditions. Set $G_{t}=(0,0, t)+G, t \in \mathbb{R}$. For any $t<0, G_{t} \cap S=\emptyset$, and if $t>0$ is large enough, $G_{t} \cap S \neq \emptyset$. Moreover, $G_{t} \cap S$ is disjoint from $\partial\left(G_{t}\right) \cup \partial(S), t \in \mathbb{R}$. Let $t_{0}=\operatorname{Infimum}\left\{t \in \mathbb{R}: G_{t} \cap S \neq \emptyset\right\}$. As $S$ and $G_{t}$ are properly immersed, it is not 
hard to see that $G_{t_{0}} \cap S \neq \emptyset$. Therefore, $G_{t_{0}}$ touches $S$ at an interior point, which contradicts the maximum principle.

A first version of this lemma, corresponding to the case where $D$ is a rectangle, was proved in [14]. As a consequence, we have

Corollary 4.3. Let $X: M \rightarrow \mathbb{R}^{3}$ be a properly immersed minimal surface of finite topology and compact boundary.

Let $W$ be a wedge as in Lemma 4.2, and suppose that $X(M) \cap \partial(W)$ lies in a solid right circular cylinder with axis parallel to $l(W)$.

Then, there exists $T>0$ such that $X^{-1}\left(\mathbb{R}^{3}-\left(W \cap\left\{x_{3}=t\right\}\right)\right)$ has a finite number of components, $t \geq T$.

Moreover, if $t \geq T$ and $\Omega_{t}$ is any connected component of $X^{-1}\left(W \cap\left\{x_{3} \geq t\right\}\right)$, then $X\left(\Omega_{t}\right)$ satisfies:

- It is a graph homeomorphic to a closed half plane.

- It is contained in a slab, and so the Gauss map of $X\left(\Omega_{t}\right)$ uniformly converges, as the distance to $l(W)$ goes to infinity, to the normal vector of the slab.

In particular, $X^{-1}\left(W \cap\left\{x_{3}=t\right\}\right)$ consists of a finite set of pairwise disjoint divergent simple arcs.

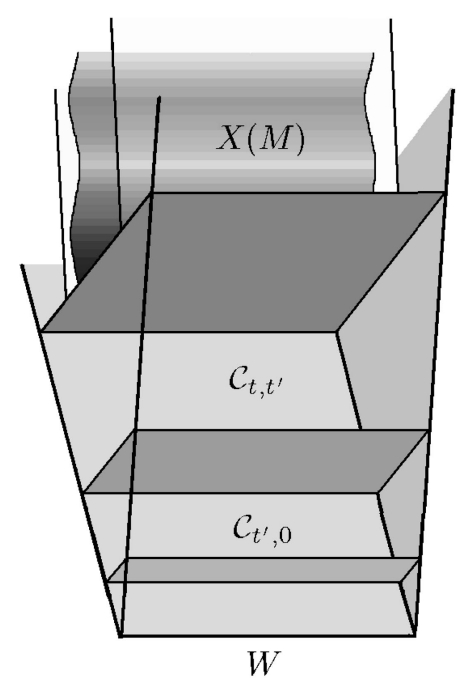

FiguRE $10 . \mathcal{C}_{t^{\prime}, 0}$ and $\mathcal{C}_{t, t^{\prime}}$

Proof. Take $t_{0}>0$ large enough so that $\left\{x_{3} \geq t_{0}\right\} \cap X(\partial(M))=\emptyset$. Then, up to the translation $x \rightarrow x-\left(0,0, t_{0}\right)$, we have $X_{3}(\partial(M)) \subset\left\{x_{3}<0\right\}$. For simplicity, the new wedge $\left(0,0,-t_{0}\right)+W$ will be also denoted by $W$. Denote by $\Pi_{1}$ and $\Pi_{2}$ the two half planes in $\partial(W)$.

For any $t>t^{\prime} \geq 0$ consider the solid right cylinder $\mathcal{C}_{t, t^{\prime}} \stackrel{\text { def }}{=}\left\{t^{\prime} \leq x_{3} \leq t\right\} \cap W$ over the quadrilateral $D_{t, t^{\prime}}=\left\{x_{2}=0\right\} \cap \mathcal{C}_{t, t^{\prime}}$. We label by $A_{1}^{t, t^{\prime}}, C_{1}^{t, t^{\prime}}, A_{2}^{t, t^{\prime}}$ and $C_{2}^{t, t^{\prime}}$ the edges of $D_{t, t^{\prime}}$ lying in $\left\{x_{3}=t\right\}, \Pi_{1},\left\{x_{3}=t^{\prime}\right\}$ and $\Pi_{2}$, respectively. If $t^{\prime}$ 
and $t-t^{\prime}$ are large enough, the lengths of these edges satisfy

$$
\left|A_{1}^{t^{\prime}, 0}\right|+\left|A_{2}^{t^{\prime}, 0}\right|<\left|C_{1}^{t^{\prime}, 0}\right|+\left|C_{2}^{t^{\prime}, 0}\right|, \quad\left|A_{1}^{t, t^{\prime}}\right|+\left|A_{2}^{t, t^{\prime}}\right|<\left|C_{1}^{t, t^{\prime}}\right|+\left|C_{2}^{t, t^{\prime}}\right| .
$$

By Lemma 4.3 the image under $X$ of any connected component of $X^{-1}\left(\mathcal{C}_{t^{\prime}, 0}\right)$ meets $D_{t^{\prime}, 0}$, and the same holds for $X^{-1}\left(\mathcal{C}_{t, t^{\prime}}\right)$ and $D_{t, t^{\prime}}$. Since $X$ is proper and $D_{t^{\prime}, 0} \cup D_{t, t^{\prime}}$ is compact, we deduce that $X^{-1}\left(\mathcal{C}_{t^{\prime}, 0}\right)$ and $X^{-1}\left(\mathcal{C}_{t, t^{\prime}}\right)$ have a finite number of connected components. Since any connected component of

$$
X^{-1}\left(\mathbb{R}^{3}-\left(W \cap\left\{x_{3}=t^{\prime}\right\}\right)\right)
$$

meets either $X^{-1}\left(\mathcal{C}_{t^{\prime}, 0}\right)$ or $X^{-1}\left(\mathcal{C}_{t, t^{\prime}}\right)$, the first part of the corollary follows for any $T \geq t^{\prime}$. See Figure 10

For the second part, note that no properly immersed annular end lies in a wedge (see [10] or Theorem 2.4). Therefore, there exists $T_{0}$ large enough so that $M_{t} \stackrel{\text { def }}{=}$ $X^{-1}\left(\mathbb{R}^{3}-\left(W \cap\left\{x_{3} \geq t\right\}\right)\right)$ consists of a finite collection of simply connected domains bounded by a finite number of simple arcs, $t \geq T_{0}$. Take a compact subset $K \subset M$ such that $M_{T_{0}}-\stackrel{\circ}{K}$ is the union of a finite number of domains homeomorphic to a closed half plane. Then, choose $T>T_{0}$ in such a way that $K \cap M_{T}=\emptyset$. From Theorem 2.2 it is not hard to see that $M_{t}$ consists of a finite collection of simply connected domains homeomorphic to a closed half plane, $t \geq T$. Using Lemma 4.2 we obtain the desired conclusion.

Now we can prove the main result of this subsection.

Theorem 4.2. Let $\Pi_{1}, \Pi_{2}$ and $\Pi_{3}$ be three pairwise non-parallel and disjoint closed half planes in $\mathbb{R}^{3}$ satisfying $\partial\left(\Pi_{j}\right) \| \partial\left(\Pi_{h}\right), h \neq j$, and $\overline{\mathcal{E}\left(\bigcup_{j=1}^{3} \Pi_{j}\right)}=\mathbb{R}^{3}$.

Let $X: M \rightarrow \mathbb{R}^{3}$ be a properly immersed minimal surface of finite topology, and suppose that $X(M) \cap\left(\bigcup_{j=1}^{3} \Pi_{j}\right)=\emptyset$.

Then, $X$ has finite total curvature, and all its ends are asymptotic to planes.

Proof. By standard arguments, it suffices to prove the theorem for a properly immersed minimal annulus $\left.\left.X: A \rightarrow \mathbb{R}^{3}, A \cong \mathbb{S}^{1} \times\right] 0,1\right]$.

Let $\mathcal{C}$ be a solid circular cylinder containing $\partial\left(\Pi_{j}\right), j=1,2,3$. In what follows, for simplicity, the closed half plane $\overline{\Pi_{j}-\mathcal{C}}$ will be also denoted as $\Pi_{j}$.

We set $\Pi_{4}=\Pi_{1}$ and $l_{j}=\partial\left(\Pi_{j}\right) \subset \partial(\mathcal{C}), j=1,2,3,4$. Up to relabeling, $W_{j} \stackrel{\text { def }}{=}$ $\mathcal{E}\left(\Pi_{j} \cup \Pi_{j+1}\right)$ is a truncated wedge, $W_{j} \cap\left(\bigcup_{j=1}^{3} \Pi_{j}\right)=\Pi_{j} \cup \Pi_{j+1}$ and $a\left(W_{j}\right)<\pi$, $j=1,2,3$. Without loss of generality, we can suppose that $X(\partial(A)) \cap W_{j}=\emptyset$, $j=1,2,3$. Denote by $S_{j}$ the strip $\mathcal{E}\left(l_{j} \cup l_{j+1}\right) \subset \partial\left(W_{j}\right)$, by $\Sigma_{j}$ the plane containing $S_{j}$, and by $v_{j}$ the normal vector of $\Sigma_{j}$ pointing to $W_{j}, j=1,2,3$. We also denote by $\Sigma_{j}^{t}$ the plane $t v_{j}+\Sigma_{j}$, and by $W_{j}^{t}$ the truncated wedge $W_{j} \cap\left(\bigcup_{s \geq t} \Sigma_{j}^{s}\right), t \in \mathbb{R}$, $j=1,2,3$.

From Corollary 4.3, there is $T>0$ large enough so that $X(M) \cap\left(\bigcup_{j=1}^{3} W_{j}^{T}\right)$ consists of a finite set of graphs homeomorphic to a closed half plane and lying in slabs. Let $\Sigma$ be an arbitrary plane meeting the polyhedral cylinder $\overline{\mathbb{R}^{3}-\bigcup_{j=1}^{3} W_{j}^{T}}$ in a compact set. By Corollary $4.2, X^{-1}(\Sigma)$ contains finitely many divergent simple arcs, and so $\Sigma$ is a finite plane for $X$. From Corollary [3.1] (or Lemma [3.1] and the Mo-Osserman theorem [22]), we get that $X$ has finite total curvature. 
Finally, Osserman's theorem implies that $A$ is conformally equivalent to a compact once-punctured disc, and the Gauss map extends meromorphically to the puncture. From Lemma 4.2, $X(A)$ lies in a slab, and so $X(A)$ is a planar end.

Corollary 4.4. Let $\Pi_{1}, \Pi_{2}, \Pi_{3}$ and $X: M \rightarrow \mathbb{R}^{3}$ be as in Theorem 4.2 Assume that $M$ has only one end and $\partial(M)=\emptyset$.

Then, $X(M)$ is a plane.

Proof. Take into account that the only complete minimal surfaces with empty boundary, finite total curvature and only one planar end are planes.

4.3. Minimal surfaces of bounded curvature. In this subsection we improve Theorem 4.2 and Corollary 4.4 for minimal surfaces of bounded curvature. As we will see later in Remark 4.1, this hypothesis is fundamental. We also deal with the problem of deciding whether a properly immersed minimal surfaces has finite type or not.

The following notation will be required:

Let $X: M \rightarrow \mathbb{R}^{3}$ be a properly immersed minimal surface of finite topology. Denote by $(\eta, g)$ the Weierstrass data of $X$. Remember that $X=\operatorname{Re} \int\left(\Phi_{1}, \Phi_{2}, \Phi_{3}\right)$, where $\Phi_{1}=\frac{1}{2}\left(1-g^{2}\right) \eta, \Phi_{2}=\frac{i}{2}\left(1+g^{2}\right) \eta$ and $\Phi_{3}=g \eta$. Following [24], $X$ is said to be of finite type if $M$ is conformally equivalent to a compact Riemann surface with compact boundary punctured in a finite set of interior points, and the 1-forms $\frac{d g}{g}$ and $\Phi_{3}$ extend meromorphically to the punctures.

Theorem 4.3. Let $\Pi_{1}, \Pi_{2}$ and $\Pi_{3}$ be three pairwise non-parallel and disjoint closed half planes in $\mathbb{R}^{3}$ such that $\partial\left(\Pi_{j}\right) \| \partial\left(\Pi_{h}\right), h \neq j$, and $\overline{\mathcal{E}\left(\bigcup_{j=1}^{3} \Pi_{j}\right)}=\left\{x_{3} \geq 0\right\}$.

Let $X: M \rightarrow \mathbb{R}^{3}$ be a properly immersed minimal surface of finite topology such that $X(M) \cap\left(\bigcup_{j=1}^{3} \Pi_{j}\right)=\emptyset$.

Then, $M$ is parabolic. Furthermore, if $X$ has bounded curvature, then $X$ has finite total curvature.

Proof. It suffices to prove the theorem for a properly immersed minimal annulus $\left.\left.X: A \rightarrow \mathbb{R}^{3}, A \cong \mathbb{S}^{1} \times\right] 0,1\right]$.

Let $\mathcal{C}$ be a solid circular cylinder containing $\bigcup_{i=1}^{3} \partial\left(\Pi_{i}\right)$, and for simplicity, label $\overline{\Pi_{j}-\mathcal{C}}$ as $\Pi_{j}, j=1,2,3$. Denote $l_{j}=\partial\left(\Pi_{j}\right) \subset \partial(\mathcal{C}), j=1,2,3$. Up to relabeling, we can suppose that $W_{j} \stackrel{\text { def }}{=} \mathcal{E}\left(\Pi_{j} \cup \Pi_{j+1}\right)$ is a truncated wedge of angle less than $\pi$, and $W_{j} \cap\left(\bigcup_{j=1}^{3} \Pi_{j}\right)=\Pi_{j} \cup \Pi_{j+1}, j=1,2$. Therefore, $\Pi_{1}$ and $\Pi_{3}$ lie in horizontal planes.

Moreover, take $\mathcal{C}$ of large enough radius in such a way that $X(\partial(A)) \cap W_{j}=\emptyset$, $j=1,2$.

Denote by $S_{j}$ the $\operatorname{strip} \mathcal{E}\left(l_{j} \cup l_{j+1}\right) \subset \partial\left(W_{j}\right)$, by $\Sigma_{j}$ the plane containing $S_{j}$, and by $v_{j}$ the normal vector of $\Sigma_{j}$ pointing to $W_{j}, j=1,2$. Denote by $\Sigma_{j}^{t}$ the plane $t \cdot v_{j}+\Sigma_{j}$, and by $W_{j}^{t}$ the truncated wedge $W_{j} \cap\left(\bigcup_{s \geq t} \Sigma_{j}^{s}\right), t \in \mathbb{R}, j=1,2$.

Reasoning as in the proof of Theorem 4.2, there is $T>0$ large enough so that $X(A) \cap\left(W_{1}^{T} \cup W_{2}^{T}\right)$ consists of a finite set of graphs lying in slabs and homeomorphic to a closed half plane. Moreover, thanks to Lemma 4.2, we can find a large enough $t_{0}>0$ such that $(i) \bigcup_{j=1}^{2}\left(\Sigma_{j}^{T} \cap W_{j}\right) \subset\left\{x_{3}<t_{0}\right\}$; and $(i i) X(A) \cap\left(\left\{x_{3} \geq t_{0}\right\}\right)$ consists of a finite number (maybe zero) of graphs lying in non-horizontal slabs and 
homeomorphic to a closed half plane. In particular, $\left\{x_{3}=t_{0}\right\}$ is a finite plane, and from [17] we infer that $M$ is parabolic.

Assume now that, in addition, $X$ has bounded curvature. Since $X_{3}^{-1}\left(t_{0}\right)$ consists of a finite set of curves, it is not hard to check that $\phi_{3}$ extends meromorphically to the end. Hence, $X$ is of finite type (see Xavier [25] and [23]). Summarizing, $A$ is conformally equivalent to an once-punctured closed disc: $A \equiv \overline{\mathbb{D}}^{*} \stackrel{\text { def }}{=} \overline{\mathbb{D}}-\{0\}, \eta g$ extends meromorphically to 0 , and $g=P e^{Q}$, where $P$ and $Q$ extend meromorphically to 0 too.

Reasoning by contradiction, suppose that $X$ does not have finite total curvature. Thus, $Q$ has a pole of order $k>0$ at the end, and so there are $2 k$ divergent Julia rays $\left\{r_{1} \ldots, r_{2 k}\right\}$ in $\overline{\mathbb{D}}^{*}$ meeting at equal angles at 0 (up to a biholomorphism and up to removing a compact piece of $A$ if necessary, we will suppose that $r_{i}$ is a segment joining 0 and $\partial(\mathbb{D}), i=1, \ldots, 2 k$, that is to say, $\left.Q(z)=c z^{-k}, c \in \mathbb{C}^{*}\right)$. This means that $g$ has a well-defined limit, as $z \rightarrow 0$, on radial closed sectors of $\overline{\mathbb{D}}^{*}$ contained in $\overline{\mathbb{D}}^{*}-\bigcup_{j=1}^{2 k} r_{j}$, and this limit is equal to either 0 or $\infty$. From Lemma $4.2(b)$, there are no connected components of $X^{-1}\left(\left\{x_{3} \geq t_{0}\right\}\right)$ whose image under $X$ lies in non-horizontal slabs (the only asymptotic values for $g$ are 0 and $\infty$ ), and so $X(A) \subset\left\{x_{3}<t_{0}\right\}$.

Since $X$ does not have finite total curvature, Theorem 4.1 (or the cone lemma 10]) implies that $X(A)$ does not lie in a slab. Thus, the third coordinate function is proper, and, up to scaling and removing a compact subset of $A$,

$$
A \equiv \overline{\mathbb{D}}-\{0\} \quad \text { and } \quad \eta g=\frac{1}{z} .
$$

Since the Gauss curvature is given by

$$
K(z)=-\left(\frac{4|\mathrm{~d} \log (g)|}{|\eta g|\left(\frac{1}{|g|}+|g|\right)^{2}}\right)^{2}=-\left(\frac{4|z|\left|\frac{P^{\prime}}{P}+Q^{\prime}\right||d z|}{\left(\frac{1}{|g|}+|g|\right)^{2}}\right)^{2}, z \in \overline{\mathbb{D}}^{*},
$$

then $K$ is not bounded on the non-compact set $|g|^{-1}(1)$, which is absurd and proves the theorem.

Corollary 4.5. Let $\Pi_{1}, \Pi_{2}, \Pi_{3}$, and $X: M \rightarrow \mathbb{R}^{3}$ be as in Theorem 4.3. Assume that $\partial(M)=\emptyset, M$ has only one end, and $X$ has bounded curvature.

Then, $X(M)$ is a plane.

Proof. From Theorem $4.3 X$ has finite total curvature. We can suppose that $\Pi_{1}$ and $\Pi_{3}$ are horizontal planes, and define the truncated wedges $W_{1}$ and $W_{2}$ as in the proof of that theorem.

The result is clear if $X(M)$ lies in a horizontal half space.

Assume that $X(M)$ does not lie in a horizontal half space. Therefore, reasoning as in the proof of Theorem 4.2. $X(M) \cap\left(W_{1} \cup W_{2}\right)$ contains a properly immersed closed half plane satisfying the hypothesis of Lemma 4.2

Hence, $X(M)$ contains a graph over a half plane lying in a slab. Since $X$ has finite total curvature, it is not hard to check that the unique end of $X(M)$ is planar, and so $X(M)$ is a plane.

An interesting problem is to decide whether a minimal surface is of finite type or not. We are going to answer this question when the surface has bounded curvature and the level curves associated to three parallel planes lie in some special planar domains (which we will call city maps). The following notation is required. 
Let $\left\{l_{1}, \ldots, l_{r}\right\}$ be a finite set of pairwise disjoint half lines in a plane $\Pi$ of $\mathbb{R}^{3}$, and let $B$ be an open Euclidean ball in $\Pi$ such that $B \cap l_{j} \neq \emptyset, j=1, \ldots, r$. Let $\mathcal{L}$ denote the set $\left\{\left(l_{1} \ldots, l_{r}\right), B\right\}$, and let $|\mathcal{L}|=B \cup\left(\bigcup_{l \in \mathcal{L}} l\right)$. Let $\tau_{\mathcal{L}}$ denote the number

$$
\tau_{\mathcal{L}}=\operatorname{Minimum}\left\{d\left(l_{j}, l_{h}\right): l_{j} \| l_{h}\right\}
$$

where $d$ means Euclidean distance, and take $\epsilon \in] 0, \tau_{\mathcal{L}}\left[\right.$. If $\left\{l_{1}, \ldots, l_{r}\right\}$ does not contain any pair of parallel half lines, then $\tau_{\mathcal{L}}=+\infty$ and $\epsilon$ can be any positive real number. Then, set

$$
\mathcal{N}(\mathcal{L}, \epsilon)=\left\{P \in \Pi: \mathrm{d}(P,|\mathcal{L}|)<\frac{\epsilon}{2}\right\}
$$

By definition, $\mathcal{N}(\mathcal{L}, \epsilon)$ is said to be the city map of radius $\epsilon$ associated to $\mathcal{L}$.

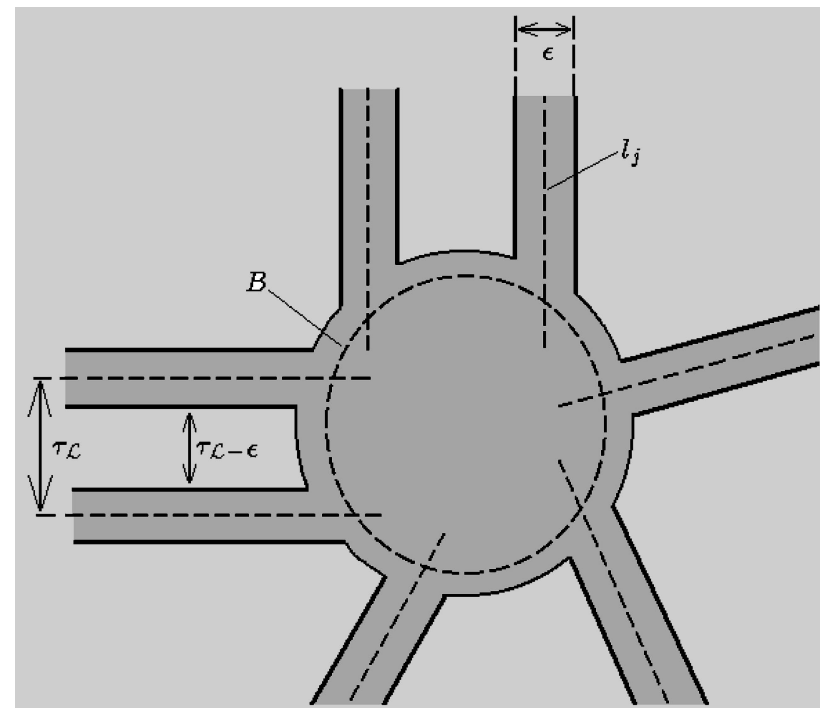

Figure 11. A city map $\mathcal{N}(\mathcal{L}, \epsilon)$.

Let $\mathcal{L}=\left(\left\{l_{1}, \ldots, l_{r}\right\}, B\right) \subset \Pi$ and $\mathcal{L}^{\prime}=\left(\left\{l_{1}^{\prime}, \ldots, l_{r}^{\prime}\right\}, B^{\prime}\right) \subset \Pi^{\prime}$ as above. We say that the city maps $\mathcal{N}(\mathcal{L}, \epsilon)$ and $\mathcal{N}\left(\mathcal{L}^{\prime}, \epsilon^{\prime}\right)$ are parallel if $\Pi \| \Pi^{\prime}, \mathfrak{p}\left(B^{\prime}\right)=B$ and $\mathfrak{p}\left(l_{j}^{\prime}\right)=l_{j}, j=1, \ldots, r$, where $\mathfrak{p}$ is the orthogonal projection on the plane $\Pi$.

Theorem 4.4. Let $\mathcal{N}_{j} \equiv \mathcal{N}\left(\mathcal{L}_{j}, \epsilon_{j}\right), j=1,2,3$, be three parallel city maps, and let $\Pi_{j}$ be the plane containing $\mathcal{N}_{j}, j=1,2,3$. Assume that $\Pi_{2}$ lies in the slab bounded by $\Pi_{1}$ and $\Pi_{3}$, and that

$$
\epsilon_{2} \epsilon_{j}<d\left(\Pi_{j}, \Pi_{2}\right)^{2}<\left(\tau-\epsilon_{2}\right)\left(\tau-\epsilon_{j}\right), \quad j=1,3,
$$

where $\tau=\tau_{\mathcal{L}_{j}}, j=1,2,3$.

Let $X: M \rightarrow \mathbb{R}^{3}$ be a properly immersed minimal surface of finite topology satisfying $X(M) \cap\left(\bigcup_{j=1}^{3} \Pi_{j}\right) \subset \bigcup_{j=1}^{3} \mathcal{N}_{j}$.

Then, $M$ is parabolic. Moreover, if $X$ has bounded curvature, then $X$ is of finite type.

Proof. It suffices to check the theorem for a properly immersed minimal annulus $\left.\left.X: A \rightarrow \mathbb{R}^{3}, A \cong\right] 0,1\right] \times \mathbb{S}^{1}$. 
Suppose that $X(A)$ is not a piece of a plane. For the sake of simplicity, write $\delta_{j}=d\left(\Pi_{j}, \Pi_{2}\right), j=1,3$. Denote by $\mathcal{S}, \mathcal{S}_{1}$ and $\mathcal{S}_{3}$ the slabs bounded by $\Pi_{1} \cup \Pi_{3}$, $\Pi_{1} \cup \Pi_{2}$ and $\Pi_{3} \cup \Pi_{2}$, respectively.

Let $\mathfrak{p}$ denote the orthogonal projection on $\Pi_{2}$. Write $\mathcal{L}_{j}=\left\{\left(l_{1}^{j}, \ldots, l_{r}^{j}\right), B_{j}\right\}$, $j=1,2,3$, where $\mathfrak{p}\left(l_{h}^{j}\right)=l_{h}^{2}, h=1, \ldots, r, \mathfrak{p}_{3}\left(B_{j}\right)=B_{2}, j=1,3$. Let $\mathcal{C}$ denote a solid circular cylinder orthogonal to $\Pi_{2}$ and satisfying $\mathcal{C} \cap \Pi_{j}=B_{j}, j=1,2,3$. Up to enlarging the radius of the squares $B_{j}$ of the city maps, we can assume that $X(\partial(A)) \subset \mathcal{C}$.

We are going to prove that $\Pi_{2}$ is a finite plane for $X$. It is sufficient to check that for $\mathcal{C}$ of large enough radius, any component of $X^{-1}\left(\mathbb{R}^{3}-\Pi_{2}\right)$ meets the compact set $X^{-1}(\mathcal{C} \cap \mathcal{S})$.

Let $T_{1}^{j}, \ldots, T_{r}^{j}$ denote the connected components of $\Pi_{j}-\mathcal{N}_{j}, j=1,2,3$, where $\mathfrak{p}\left(T_{h}^{j}\right) \cap T_{h}^{2} \neq \emptyset, h=1, \ldots, r, j=1,3$. Up to adding a suitable set of half lines to $\left\{l_{1}^{j}, \ldots, l_{r}^{j}\right\}, j=1,2,3$, and without loss of generality, we will suppose that $S_{h}^{j} \stackrel{\text { def }}{=} \mathcal{E}\left(T_{j}^{h}\right)$ is a truncated planar sector in $\Pi_{j}$ of angle $a\left(S_{h}^{j}\right)<\pi, h=1, \ldots, r$, $j=1,2,3$. Denote by $C_{h}^{j}$ the truncated tetrahedral domain $\mathcal{E}\left(S_{h}^{j} \cup S_{h}^{2}\right)$, and by $F^{+}\left(C_{h}^{j}\right)$ and $F^{-}\left(C_{h}^{j}\right)$ the two non-compact faces of $C_{h}^{j}$ not contained in $\Pi_{2} \cup \Pi_{j}$, $h=1, \ldots, r, j=1,3$.

Let $C$ be a truncated tetrahedral domain satisfying the hypothesis of Theorem 2.5. (b). Up to a homothety, suppose $\delta_{j}<1$, and note that $\mathfrak{h}(C)=1>\delta_{j}, j=1,3$. Moreover, take $C$ in such a way that $0<\varrho(C)<a\left(S_{h}^{i}\right)$, for any $h \in\{1, \ldots, r\}$ satisfying $a\left(S_{h}^{i}\right)>0, i=1,2,3$. If the radius of $B_{j}$ (and so the radius of $\mathcal{C}$ ) is large enough, $j=1,2,3$, there exists a rigid motion $R_{h}^{j}$ such that

$$
\left(F_{1}(C) \cup F_{2}(C)\right) \cap R_{h}^{j}\left(C_{h}^{j}\right)=\emptyset, \quad R_{h}^{j}\left(F^{+}\left(C_{h}^{j}\right) \cup F^{-}\left(C_{h}^{j}\right)\right) \cap C=\emptyset,
$$

and the faces $R_{h}^{j}\left(F^{+}\left(C_{h}^{j}\right)\right)$ and $R_{h}^{j}\left(F^{-}\left(C_{h}^{j}\right)\right)$ lie in distinct connected components of $R_{h}^{j}\left(C_{h}^{j}\right)-C, j=1,3$ (see Figure 12). Hence, thanks to Theorem 2.5 there is no connected properly immersed non-flat minimal surface in $C_{h}^{j}$ with planar boundary lying in $F^{+}\left(C_{h}^{j}\right) \cup F^{-}\left(C_{h}^{j}\right), j=1,3$, for any $h$ such that $a\left(S_{h}^{i}\right)>0, i=1,2,3$.

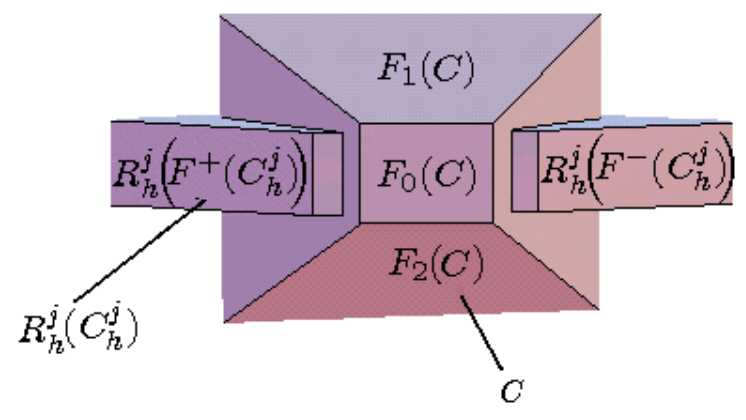

Figure 12. $C$ and $R_{h}^{j}\left(C_{h}^{j}\right)$.

Since $X(A) \cap\left(\bigcup_{j=1}^{3} \Pi_{j}\right) \subset \bigcup_{j=1}^{3} \mathcal{N}_{j}$, the above arguments imply that the image under $X$ of any connected component of $X^{-1}\left(C_{h}^{j}\right)$ intersects $\mathcal{C} \cap \mathcal{S}, j=1,3$, provided that $a\left(S_{h}^{i}\right)>0, i=1,2,3$. 
Let us see that the same holds when $a\left(S_{h}^{i}\right)=0, i=1,2,3$. In this case, $C_{h}^{j}$ is a truncated solid right cylinder over a quadrilateral, $j=1,3$. Thanks to Lemma 4.3. we know that there is no connected properly immersed non-flat minimal surface in $C_{h}^{j}$ with boundary lying in $F^{+}\left(C_{h}^{j}\right) \cup F^{-}\left(C_{h}^{j}\right)$, provided that

$$
2 \sqrt{\delta_{j}^{2}+\left(\frac{\epsilon_{j}-\epsilon_{2}}{2}\right)^{2}}<2 \tau-\epsilon_{j}-\epsilon_{2}, \quad j=1,3 ;
$$

that is to say, $\delta_{j}^{2}<\left(\tau-\epsilon_{2}\right)\left(\tau-\epsilon_{j}\right), j=1,3$, which holds from our assumptions. Therefore, taking into account that $X(A) \cap\left(\bigcup_{j=1}^{3} \Pi_{j}\right) \subset \bigcup_{j=1}^{3} \mathcal{N}_{j}$ once again, we infer that the image under $X$ of any connected component of $X^{-1}\left(C_{h}^{j}\right)$ intersects $\mathcal{C} \cap \mathcal{S}$ too, $j=1,3$.

For a contradiction, let $X^{-1}\left(\mathbb{R}^{3}-\Pi_{2}\right)$ have a connected component $\Omega$ whose imaged under $X$ is disjoint from $\mathcal{C} \cap \mathcal{S}$. Taking into account the above arguments, we see that $X(\Omega)$ is in fact disjoint from

$$
G \stackrel{\text { def }}{=}(\mathcal{C} \cap \mathcal{S}) \cup\left(\bigcup_{h=1}^{r}\left(C_{h}^{1} \cup C_{h}^{3}\right)\right) .
$$

Let $\Omega_{0}$ denote a connected component of $\Omega \cap X^{-1}(\mathcal{S})$, and let $N=X\left(\Omega_{0}\right)$. Up to relabeling, we will suppose that $N \subset \mathcal{S}_{1}$. The set $\mathcal{S}_{1}-G$ consists of a finite number of pairwise disjoint solid truncated tetrahedral cylinders, and only one of them, call it $F$, contains $N$. Observe that $F$ has only one compact face lying in $\mathcal{C}$. Moreover, two opposite non-compact faces of $F$ lie in $\Pi_{2}$ and $\Pi_{1}$, and correspond to parallel streets in $\mathcal{N}_{2}$ and $\mathcal{N}_{1}$ which contain $\partial(N)$. The hypothesis of the theorem gives

$$
\epsilon_{1}+\epsilon_{2}<2 \sqrt{\delta_{1}^{2}+\left(\frac{\epsilon_{2}-\epsilon_{1}}{2}\right)^{2}},
$$

and this contradicts Lemma 4.3.

As a consequence, $\Pi_{2}$ is a finite plane, and by [17, $A$ is parabolic.

If in addition $X$ has bounded curvature, we can use ideas from [25] or [23] to infer that $X$ is of finite type, which concludes the proof.

Corollary 4.6. Let $X: M \rightarrow \mathbb{R}^{3}$ be a properly embedded simply connected minimal surface with bounded curvature. Assume that the level curves associated to three parallel planes lie in three parallel city maps as in Theorem 4.4.

Then, $X(M)$ is the helicoid.

A considerable improvement of this corollary can be found in [19].

Proof. Since $\Pi_{2}$ is a finite plane, the result follows from [23].

We finish with the following remark.

Remark 4.1. The hypothesis of having finite topology is fundamental in Theorems 3.1, 4.1 and 4.2, and in the second part of Theorems 4.3 and 4.4 Scherk singly periodic minimal surfaces are the counterexamples.

The same occurs with the hypothesis of having bounded curvature in the second part of Theorems 4.3 and 4.4. Indeed, there exists a simply connected, properly immersed and non-flat minimal surface in $\mathbb{R}^{3}$ which has no boundary, is not of finite 
type and is contained in the union of a half space and a slab orthogonal to the half space:

Consider on $\mathbb{C}-\{0\}$ the Weierstrass data $\left(g=e^{z}, \eta=\frac{d z}{z e^{z}}\right)$. It is clear that $\Phi_{1}=$ $\frac{1}{2}\left(1-g^{2}\right) \eta$ and $\Phi_{3}=g \eta$ have no real periods on $\mathbb{C}-\{0\}$. However, $\int_{0}^{2 \pi} \Phi_{2}\left(\alpha^{\prime}(t)\right) d t \neq$ 0 , where $\alpha(t)=e^{i t}$. Therefore, the associated minimal immersion determines a singly periodic minimal surface invariant under a horizontal translation $T$ parallel to the $x_{2}$-axis. The induced immersion $Y: \mathbb{C}-\{0\} \rightarrow \mathbb{R}^{3} / T, Y(z)=$ $\operatorname{Re} \int_{1}^{z}\left(\Phi_{1}, \Phi_{2}, \Phi_{3}\right)$, is proper and has two ends which correspond to the points 0 and $\infty$. The first one is of Scherk type, and it is asymptotic to a half flat cylinder in $\mathbb{R}^{3} / T$ parallel to the plane $\left\{x_{1}=0\right\}$ and lying in the half space $\left\{x_{3} \leq 0\right\}$. The second end has unbounded curvature, and it is contained in the half space $\left\{x_{3} \geq 0\right\}$. The immersion $X: \mathbb{C} \rightarrow \mathbb{R}^{3}$ given by $X(z)=Y\left(e^{z}\right)$ has the desired properties (see Figure 131).

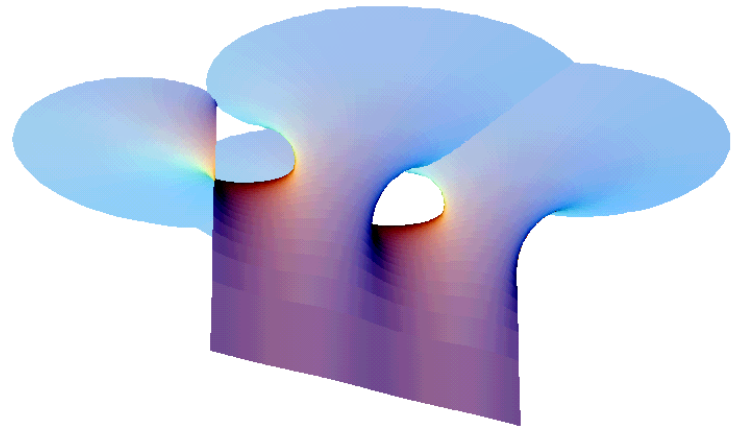

Figure $13 . \quad X(\mathbb{C})$.

On the other hand, it is well known that the only properly embedded minimal surface foliated by Jordan curves in parallel planes is the catenoid [4. The similar result for properly immersed minimal cylinders in $\mathbb{R}^{3}$ fails, as the following example shows:

Consider on $\mathbb{C}-\{0\}$ the Weierstrass data $\left(g=e^{z+\frac{a}{z}}, \eta=\frac{d z}{z g}\right)$, where $a \in \mathbb{R}$. It is clear that $\Phi_{3}=\frac{d z}{z}$ has no real periods. Moreover, by an intermediate value argument, there exists $a \in \mathbb{R}$ such that $\Phi_{1}$ and $\Phi_{2}$ have no real periods too. Hence, the minimal immersion $Y(z)=\operatorname{Re} \int_{1}^{z}\left(\Phi_{1}, \Phi_{2}, \Phi_{3}\right)$ is well defined, and its third coordinate function $Y_{3}(z)=\log (|z|)$ is proper. The two ends of $Y(\mathbb{C}-\{0\})$ lie in a half space of $\mathbb{R}^{3}$, and so they are critical from the point of view of Theorem 4.3 Of course, both ends have unbounded curvature. Hence, the hypothesis of having bounded curvature in the second part of Theorem 4.3 is fundamental even if we assume that the surface is of finite type.

\section{ACKNOWLEDGEMENTS}

The author is grateful to the referee for his helpful comments and suggestions for the revision of the paper.

\section{REFERENCES}

[1] Ahlfors, L. V., Sario, L.: Riemann surfaces. Princeton Univ. Press: Princeton, New Jersey (1960). MR 22:5729 
[2] Burckel, R.: An introduction to complex analysis. Vol. 1, Birkhäuser Verlag, Basel und Stuttgart (1979). MR 81d:30001

[3] Choe, J., Soret, M.: Nonexistence of certain complete minimal surfaces with planar ends. Comment. Math. Helv., Vol 75 (2000), 189-199. MR 2002a:53007

[4] Collin, P.: Topologie et courbure des surfaces minimales propement plongées de $\mathbb{R}^{3}$. Ann. Math., 145 (1997), 1-31. MR 98d:53010

[5] Colding, Tobias H., Minicozzi, William P.: Complete properly embedded minimal surfaces in $\mathbb{R}^{3}$. Duke Math. J., 107 (2001), 421-426. MR 2002a:53008

[6] Collin, P., Kusner, R., Meeks, W. H. III, Rosenberg, H.: The topology, geometry and conformal structures of properly embedded minimal surfaces. Preprint.

[7] Fang, Y., Meeks, W. H., III: Some global properties of complete minimal surfaces of finite topology in $\mathbb{R}^{3}$. Topology, 30 (1991), 9-20. MR 92g:53008

[8] Goluzin, G. M.: Geometric theory of functions of a complex variable. Translations of Mathematical Monographs, Vol. 26, American Math. Soc., Providence, Rhode Island 02904, (1969). MR 40:308

[9] Hoffman, D., Meeks, W. H., III: The asymptotic behavior of properly embedded minimal surfaces of finite topology. J. Am. Math. Soc., 2 (1989), 667-681. MR 90f:53010

[10] Hoffman, D., Meeks, W.H., III: The strong half space theorem for minimal surfaces. Inventiones Math., 101 (1990), 373-377. MR 92e:53010

[11] Jenkins, H., Serrin, J.: Variational problems of minimal surface type. II. Boundary value problems for the minimal surface equation. Arch. Rat. Mech. Anal., 21, (1966), 321-342. MR 22:8221

[12] Jorge, L. P. M., Meeks, W. H., III: The topology of complete minimal surfaces of finite total Gaussian curvature. Topology, 2 (1983), 203-221. MR 84d:53008

[13] Korevaar, N. J., Kusner, R., Solomon, B.: The structure of complete embedded surfaces with constant mean curvature, J. Differential Geom., 30 (1989), 465-503. MR 90g:53011

[14] López, F. J., Martín, F.: Minimal surfaces in a wedge of a slab. Comm. in Ann. and Geom., 9 (2001), 683-723. MR 2002k:53013

[15] López, F. J.: Minimal surfaces in a cone. Annals of Global Analysis and Geometry, 20 (2001), 253-299. MR 2003c:53015

[16] López, F. J., Pérez, J.: Parabolicity and Gauss map of minimal surfaces. To appear in Indiana J. Math.

[17] Meeks, W. H., III, and Rosenberg, H.: The geometry and conformal structure of properly embedded minimal surfaces of finite topology in $\mathbb{R}^{3}$. Invent. Math., 114 (1993), 625-639. MR 94i:53003

[18] Meeks, W. H., III, Rosenberg, H.: Maximum principles at infinity with applications to minimal and constant mean curvature surfaces. Preprint.

[19] Meeks, W. H., III, Rosenberg, H.: The uniqueness of the helicoid and the asymptotic geometry of properly embedded minimal surfaces with finite topology. Preprint.

[20] Morales, S.: On the existence of a proper minimal surface in $\mathbb{R}^{3}$ with the conformal type of a disk. To appear in GAFA.

[21] Osserman, R.: A Survey of Minimal Surfaces. Vol. 1, Cambridge Univ. Press, New York (1989). MR 41:934 (1st ed.)

[22] Mo, X., Osserman, R.: On the Gauss map and total curvature of complete minimal surfaces and an extension of Fujimoto's theorem. J. Differential Geom., 31 (1990), 343-355. MR 91a:53013

[23] Rodriguez, L., Rosenberg, H.: Minimal surfaces in $\mathbb{R}^{3}$ with one end and bounded curvature. Manuscripta-Math., 96 (1998), 3-7. MR 99c:53010

[24] Rosenberg, H.: Minimal surfaces of finite type. Bull. Soc. Math. France, 123 (1995), 351359. MR 97a:53011

[25] Xavier, F.: Why no new complete simply-connected embedded minimal surfaces have been found since 1776. Preprint.

Departamento de Geometría y Topología, Universidad de Granada, 18071 Granada, SPAIN

E-mail address: fjlopez@goliat.ugr.es 